\title{
LINES OF MINIMA AND TEICHMÜLLER GEODESICS
}

\author{
YOUNG-EUN CHOI, KASRA RAFI, AND CAROLINE SERIES
}

\begin{abstract}
For two measured laminations $\nu^{+}$and $\nu^{-}$that fill up a hyperbolizable surface $S$ and for $t \in(-\infty, \infty)$, let $\mathcal{L}_{t}$ be the unique hyperbolic surface that minimizes the length function $e^{t} l\left(\nu^{+}\right)+e^{-t} l\left(\nu^{-}\right)$on Teichmüller space. We characterize the curves that are short in $\mathcal{L}_{t}$ and estimate their lengths. We find that the short curves coincide with the curves that are short in the surface $\mathcal{G}_{t}$ on the Teichmüller geodesic whose horizontal and vertical foliations are respectively, $e^{t} \nu^{+}$and $e^{-t} \nu^{-}$. By deriving additional information about the twists of $\nu^{+}$and $\nu^{-}$around the short curves, we estimate the Teichmüller distance between $\mathcal{L}_{t}$ and $\mathcal{G}_{t}$. We deduce that this distance can be arbitrarily large, but that if $S$ is a once-punctured torus or four-times-punctured sphere, the distance is bounded independently of $t$.
\end{abstract}

\section{INTRODUCTION}

Suppose that $\nu^{+}$and $\nu^{-}$are measured laminations which fill up a hyperbolizable surface $S$. The object of this paper is to compare two paths in the Teichmüller space $\mathcal{T}(S)$ of $S$ determined by $\nu^{+}$and $\nu^{-}$. The first is the Teichmüller geodesic $\mathcal{G}=\mathcal{G}\left(\nu^{+}, \nu^{-}\right)$, whose time $t$ Riemann surface $\mathcal{G}_{t} \in \mathcal{G}$ supports a quadratic differential $q_{t}$ whose horizontal and vertical foliations are $\nu_{t}^{+}=e^{t} \nu^{+}$and $\nu_{t}^{-}=e^{-t} \nu^{-}$, respectively [7]. It is characterized as the locus where the product of the extremal lengths $E\left(\nu^{+}\right) E\left(\nu^{-}\right)$attains its minimum value. The second is the Kerckhoff line of minima $\mathcal{L}=\mathcal{L}\left(\nu^{+}, \nu^{-}\right)$[11]. At time $t$, $\mathcal{L}_{t} \in \mathcal{L}$ is the unique hyperbolic surface that minimizes the length function $l\left(\nu_{t}^{+}\right)+l\left(\nu_{t}^{-}\right)=e^{t} l\left(\nu^{+}\right)+e^{-t} l\left(\nu^{-}\right)$on $\mathcal{T}(S)$. Lines of minima have many properties in common with Teichmüller geodesics, see [11], and have been shown to be closely linked to deforming Fuchsian into quasifuchsian groups by bending, see [24].

Part of the motivation for studying this question stems from a central ingredient of the proof of the ending lamination theorem [4]. Suppose that $N$ is a hyperbolic 3-manifold homeomorphic to $S \times \mathbb{R}$. The ending lamination theorem states that $N$ is completely determined by the asymptotic invariants of its two ends. A key step is to show that if these end invariants are induced by the laminations $\nu^{+}$and $\nu^{-}$, then 
the curves on $S$ which have short geodesic representatives in $N$ have a combinatorial characterization in terms of the complex of curves of $S$. Roughly speaking, a curve is short in $N$ if and only if the distance between the projections of $\nu^{+}$and $\nu^{-}$to some subsurface $Y \subset S$ is large in the curve complex of $Y$.

Rafi [20] found a similar combinatorial characterization which shows that these curves are almost, but not quite, the same as those curves which become short along $\mathcal{G}\left(\nu^{+}, \nu^{-}\right)$; that is, curves which become short at some time $t$, with respect to the hyperbolic metric $g_{t}$ that uniformizes $\mathcal{G}_{t}$. One of the main results of this paper is a similar characterization of curves which become short along $\mathcal{L}\left(\nu^{+}, \nu^{-}\right)$.

We are interested in comparing the two trajectories $\mathcal{G}$ and $\mathcal{L}$, in particular, to see whether or not they remain a bounded distance apart. If both $\mathcal{G}_{t}$ and $\mathcal{L}_{t}$ are contained in the thick part of $\mathcal{T}(S)$, it is relatively easy to show that the Teichmüller distance between them is bounded, independently of $t$ (see Theorem 3.9). If, however, $\mathcal{G}_{t}$ or $\mathcal{L}_{t}$ contains short curves, then we need to compare their respective thick-thin decompositions. The essential point is to estimate the lengths of curves which are short in $g_{t}$ and those that are short in $\mathcal{L}_{t}$.

Using the results of [17] and the ideas of [20], we estimate the hyperbolic length of a short curve in $g_{t}$ as follows:

Theorem A (Theorem 5.8).

$$
\frac{1}{l_{g_{t}}(\alpha)} \asymp \max \left\{D_{t}(\alpha), \log K_{t}(\alpha)\right\} .
$$

Here, $D_{t}(\alpha)$ and $K_{t}(\alpha)$ are parametric counterparts of purely topological quantities $D(\alpha)$ and $K(\alpha)$ which are determined by the combinatorics of $\nu^{+}, \nu^{-}$, and $\alpha$. Specifically, $D(\alpha)$ is large if and only if the difference between the amounts $\nu^{+}$and $\nu^{-}$twist around $\alpha$ is large, and $K(\alpha)$ is large if and only if $\nu^{+}$and $\nu^{-}$have large relative complexity in $S \backslash \alpha$, in the sense that every essential arc or closed curve in $S \backslash \alpha$ must intersect a leaf of $\nu^{+}$or $\nu^{-}$a large number of times [20].

In comparison, we prove that the hyperbolic length of a short curve in $\mathcal{L}_{t}$ is estimated by

Theorem B (Theorem 7.13).

$$
\frac{1}{l_{\mathcal{L}_{t}}(\alpha)} \asymp \max \left\{D_{t}(\alpha), \sqrt{K_{t}(\alpha)}\right\} .
$$

The proof of Theorem B is rather lengthy and is carried out in several steps. First, we give an estimate formulated in terms of another 
geometric quantity $H_{t}(\alpha)$ that satisfies $H_{t}(\alpha) \succ K_{t}(\alpha)$ (Theorem 6.1):

$$
\frac{1}{l_{\mathcal{L}_{t}}(\alpha)} \asymp \max \left\{D_{t}(\alpha), \sqrt{H_{t}(\alpha)}\right\} .
$$

In particular, it follows that a curve that is short in $g_{t}$ is at least as short in $\mathcal{L}_{t}$. In proving Theorem 6.1 , the main tool is the well-known derivative formula of Kerckhoff [10] and Wolpert [26] for the variation of length with respect to Fenchel Nielsen twist, together with the extension proved by Series [23] for variation with respect to the lengths of pants curves. Next, we show in Proposition 7.8 that on a subsurface whose injectivity radius is bounded below in $g_{t}$, the injectivity radius with respect to $\mathcal{L}_{t}$ is also bounded below, perhaps by a smaller constant. This sets up a one-to-one correspondence between these suitably defined thick components of $g_{t}$ and $\mathcal{L}_{t}$. We show moreover, in Theorem 7.10 and Corollary 7.11, that the Teichmüller distance between corresponding thick components is bounded. Finally we are able to prove that $H_{t}(\alpha) \prec K_{t}(\alpha)$, thus completing the proof of Theorem B.

In Theorem 6.2, we further estimate the twist of $\nu^{+}$and $\nu^{-}$around $\alpha$ at $\mathcal{L}_{t}$. Combined with the analogous estimate for $\mathcal{G}_{t}$ proved in [21], we are able to apply Minsky's product region theorem [19] to conclude that

Theorem C (Theorem 7.15).

$$
d_{\mathcal{T}(S)}\left(\mathcal{G}_{t}, \mathcal{L}_{t}\right)=\log \max \left\{\frac{l_{g_{t}}(\alpha)}{l_{\mathcal{L}_{t}}(\alpha)}\right\} \pm O(1),
$$

where the maximum is taken over all curves $\alpha$ that are short in $g_{t}$. Because $K_{t}(\alpha)$ can become arbitrarily large while $D_{t}(\alpha)$ remains bounded, it follows from the three theorems above that $\mathcal{G}_{t}$ and $\mathcal{L}_{t}$ do not always remain a bounded distance apart. However, in the case where $S$ is a once-punctured torus or four-times-punctured sphere, it turns out that the quantity $K_{t}(\alpha)$ is always bounded and therefore that the two paths are always within bounded distance of each other.

Prior to this paper, the only results related to the relative behavior of $\mathcal{G}$ and $\mathcal{L}$ were some partial results about their behavior at infinity. Results of Masur [14] (for Teichmüller geodesics) and of Díaz and Series [5] (for lines of minima) show that if either $\nu^{ \pm}$are supported on closed curves, or if $\nu^{ \pm}$are uniquely ergodic, then $\mathcal{G}$ and $\mathcal{L}$ limit on the same points in the Thurston boundary of $\mathcal{T}(S)$. In general, the question of the behavior at infinity remains unresolved, but see also [12] which shows that there are Teichmüller geodesics $\mathcal{G}$ which do not converge in Thurston's compactification of $\mathcal{T}(S)$. 
The paper is organized as follows. In Section 2, we recall some background facts about lines of minima and Teichmüller geodesics. In Section 3, we prove Theorem 3.9 mentioned above, which states that if both $\mathcal{G}_{t}$ and $\mathcal{L}_{t}$ are contained in the thick part of $\mathcal{T}(S)$, then the Teichmüller distance between them is bounded. By treating this special case separately in the early part of the paper, we hope to give some intuition of how we compare $\mathcal{G}_{t}, \mathcal{L}_{t}$ and what needs to be done in order to study the question in full generality. In Section 4, we review in detail the definitions of twists and Fenchel Nielsen coordinates, which we shall need in subsequent sections. In Section 5, we derive Theorem A and in addition, state the estimates for twists about short curves proved in [21]. Analogously, in Section 6, we prove Theorem 6.1 and derive estimates for twists about the short curves. Finally, in Section 7, we prove Theorems B and C. Throughout the paper, we make use of several basic length estimates on hyperbolic surfaces. The proofs, being somewhat long but relatively straightforward, are relegated to the Appendix.

\section{Preliminaries}

Throughout, $S$ is a hyperbolizable surface of finite type, possibly with punctures but with no other boundary.

2.1. Thick-thin decomposition. Let $\mathcal{S}$ denote the set of free homotopy classes of non-peripheral, non-trivial simple closed curves on $S$. If $(S, \sigma)$ is a surface with hyperbolic metric $\sigma$ and $\alpha \in \mathcal{S}$, we write $l_{\sigma}(\alpha)$ for the hyperbolic length of the unique geodesic representative of $\alpha$ with respect to $\sigma$. The Margulis constant provides a universal constant $\epsilon_{\mathcal{M}}>0$ such that all components of the $\epsilon_{\mathcal{M}}$-thin part of $(S, \sigma)$ (i.e. those parts of $S$ where the injectivity radius is less than $\epsilon_{\mathcal{M}}$ ) are horocyclic neighborhoods of cusps or annular collars about short geodesics. The $\epsilon_{\mathcal{M}}$-thick part of the surface is the complement of the thin part.

For our purposes, it is necessary to choose a constant $\epsilon_{0}>0$ sufficiently smaller than $\epsilon_{\mathcal{M}}$, in order that the $\epsilon_{0}$-thick-thin decomposition of a surface satisfies certain geometric conditions. These conditions will be mentioned when the context arises, but we assume that $\epsilon_{0}$ has been chosen once and for all so that these conditions are met. If $l_{\sigma}(\alpha)<\epsilon_{0}$, we shall say that $\alpha$ is extremely short in $\sigma$.

2.2. Notation. Since we will be dealing mainly with coarse estimates, we want to avoid keeping track of constants which are universal, in that they do not depend on any specific metric or curve under discussion. 
For functions $f, g$ we write $f \asymp g$ and $f \stackrel{*}{`} g$ to mean respectively, that there are constants $c>1, C>0$, depending only on the topology of $S$ and the fixed constant $\epsilon_{0}$, such that

$$
\frac{1}{c} g(x)-C \leq f(x) \leq c g(x)+C \text { and } \frac{1}{c} g(x) \leq f(x) \leq c g(x) .
$$

The symbols $\prec, \stackrel{*}{\prec}, \succ, \stackrel{*}{\succ}$ are defined similarly. For a positive quantity $X$, we often write $X=O(1)$ instead of $X \prec 1$ to indicate $X$ is bounded above by a constant depending only on the topology of $S$ and $\epsilon_{0}$, and more generally write $X=O(Y)$ to mean that $X / Y=O(1)$ for a positive function $Y$.

2.3. Measured laminations. We denote the space of measured laminations on $S$ by $\mathcal{M L}(S)$. Given any hyperbolic metric $\sigma$ on $S$, a measured lamination $\xi \in \mathcal{M L}(S)$ can be realized as a geodesic measured lamination with respect to $\sigma$. The hyperbolic length function extends by linearity and continuity to $\mathcal{M L}(S)$; we write $l_{\sigma}(\xi)$ for the hyperbolic length of a lamination $\xi \in \mathcal{M L}(S)$. The geometric intersection number $i(\alpha, \beta)$ of curves $\alpha, \beta \in \mathcal{S}$ also extends continuously to $\mathcal{M L}(S)$. Laminations $\mu, \nu \in \mathcal{M L}(S)$ are said to fill up $S$ if $i(\mu, \xi)+i(\nu, \xi)>0$ for all $\xi \in \mathcal{M L}(S)$. For $\xi \in \mathcal{M L}(S)$, we denote the underlying leaves by $|\xi|$.

2.4. Teichmüller space. The Teichmüller space $\mathcal{T}(S)$ of $S$ is the space of all conformal structures on $S$ up to isotopy. The Teichmüller distance $d_{\mathcal{T}(S)}\left(\Sigma, \Sigma^{\prime}\right)$ between two marked Riemann surfaces $\Sigma, \Sigma^{\prime} \in$ $\mathcal{T}(S)$ is $[\log K] / 2$, where $K$ is the smallest quasiconformal distortion of a homeomorphism from $\Sigma$ to $\Sigma^{\prime}$ which is isotopic to the identity.

Each conformal structure $\Sigma \in \mathcal{T}(S)$ is uniformized by a unique hyperbolic structure $\sigma$, and conversely, each hyperbolic structure $\sigma$ has an underlying conformal structure $\Sigma$. Thus, we also consider $\mathcal{T}(S)$ to be the space of all hyperbolic metrics on $S$ up to isotopy. The thick part $\mathcal{T}_{\text {thick }}(S)$ of $\mathcal{T}(S)$ will be defined as the subset of all hyperbolic metrics such that every closed geodesic has length bounded below by the constant $\epsilon_{0}$.

2.5. Kerckhoff lines of minima. Suppose that $\nu^{+}, \nu^{-} \in \mathcal{M L}(S)$ fill up $S$. Kerckhoff [11] showed that the length function $\sigma \mapsto l_{\sigma}\left(\nu^{+}\right)+$ $l_{\sigma}\left(\nu^{-}\right)$has a global minimum at a unique surface $\mathcal{L}_{0}$ in $\mathcal{T}(S)$. Moreover, as $t$ varies in $(-\infty, \infty)$, the minimum $\mathcal{L}_{t} \in \mathcal{T}(S)$ of $l\left(\nu_{t}^{+}\right)+l\left(\nu_{t}^{-}\right)$for the weighted laminations $\nu_{t}^{+}=e^{t} \nu^{+}$and $\nu_{t}^{-}=e^{-t} \nu^{-}$varies continuously with $t$ and traces out a path $t \mapsto \mathcal{L}_{t}$ called the line of minima $\mathcal{L}\left(\nu^{+}, \nu^{-}\right)$ of $\nu^{ \pm}$. 
2.6. Quadratic differentials. General facts about quadratic differentials and the associated metric can be found in [6, 25]. Let $\Sigma$ be a Riemann surface and $q$ a quadratic differential on $\Sigma$ which is holomorphic, except possibly at punctures, where $q$ may have a pole of order one. This ensures that the area of $\Sigma$ with respect to the area element $\left|q(z) d z^{2}\right|$ is finite, and we normalize so that the area is 1 . Let $\mathcal{Q}(\Sigma)$ be the space of all such meromorphic quadratic differentials on $\Sigma$.

The zeros and poles of $q$ are called critical points. If $z_{0}$ is not a critical point, we can define a new local coordinate

$$
w=\int_{z_{0}}^{z} \sqrt{q(z)} d z .
$$

This defines a pair of measured singular foliations on $\Sigma$; the horizontal foliation $\mathcal{H}_{q}$, along whose leaves $\operatorname{Im} w$ is constant and the vertical foliation $\mathcal{V}_{q}$, along whose leaves Re $w$ is constant. The measures on the respective foliations are determined by integrating the line element $|\sqrt{q(z)} d z|$. More precisely, for a curve $\eta$, its horizontal and vertical measures are given respectively by

$$
h_{q}(\eta)=\int_{\eta}|\operatorname{Re}[\sqrt{q(z)} d z]|, v_{q}(\eta)=\int_{\eta}|\operatorname{Im}[\sqrt{q(z)} d z]| .
$$

We call $h_{q}(\eta)$ and $v_{q}(\eta)$ respectively, the horizontal length and the vertical length of $\eta$.

Every simple closed curve $\gamma$ in $(S, q)$ has a unique $q$-geodesic representative, unless it is in a family of closed Euclidean geodesics foliating an annulus whose interior contains no singularities. We denote the $q$-geodesic length of $\gamma$ by $l_{q}(\gamma)$. It satisfies the following inequalities:

$$
\left[h_{q}(\gamma)+v_{q}(\gamma)\right] / \sqrt{2} \leq l_{q}(\gamma) \leq h_{q}(\gamma)+v_{q}(\gamma)
$$

By definition of intersection numbers for measured foliations, we have $v_{q}(\gamma)=i\left(\mathcal{V}_{q}, \gamma\right)$ and $h_{q}(\gamma)=i\left(\mathcal{H}_{q}, \gamma\right)$, so Equation(1) implies

$$
l_{q}(\gamma) \stackrel{*}{\asymp} i\left(\mathcal{V}_{q}, \gamma\right)+i\left(\mathcal{H}_{q}, \gamma\right) \text {. }
$$

This approximation will be used repeatedly.

2.7. Teichmüller geodesics. Suppose that $\Sigma, \Sigma^{\prime} \in \mathcal{T}(S)$ are marked Riemann surfaces with $d_{\mathcal{T}(S)}\left(\Sigma, \Sigma^{\prime}\right)=d$. Then there is a unique quadratic differential $q$ on $\Sigma$ such that the conformal structure on $\Sigma^{\prime}$ is obtained from that of $\Sigma$ by expanding in the horizontal direction of $q$ by a factor $e^{d}$ and contracting in the vertical direction by $e^{-d}$. The homeomorphism which realizes this is called the Teichmüller map from $\Sigma$ to $\Sigma^{\prime}$, and has quasiconformal distortion $e^{2 d}$. The 1-parameter family of quadratic differentials $q_{t}$ whose horizontal and vertical foliations are 
respectively, $e^{t} \mathcal{H}_{q}$ and $e^{-t} \mathcal{V}_{q}$, for $0 \leq t \leq d$, define the geodesic path from $\Sigma$ to $\Sigma^{\prime}$ with respect to the Teichmüller metric.

Gardiner and Masur [7] showed that for any pair of measured laminations $\nu^{+}, \nu^{-} \in \mathcal{M L}(S)$ which fill up $S$, there is a unique surface $\Sigma \in \mathcal{T}(S)$ and a unique quadratic differential $q \in \mathcal{Q}(\Sigma)$ whose horizontal and vertical foliations are $\nu^{+}, \nu^{-}$respectively. (This uses the oneto-one correspondence between the space of measured laminations and the space of measured foliations.) For $t \in \mathbb{R}$ set $\nu_{t}^{+}=e^{t} \nu^{+}, \nu_{t}^{-}=e^{-t} \nu^{-}$ and let $\mathcal{G}_{t}, q_{t}$ be the corresponding surface and quadratic differential. The path $t \mapsto \mathcal{G}_{t}$ defines a Teichmüller geodesic which we denote $\mathcal{G}=\mathcal{G}\left(\nu^{+}, \nu^{-}\right)$. We denote the hyperbolic metric on the surface $\mathcal{G}_{t} \in \mathcal{G}$ by $g_{t}$.

2.8. The balance time. Suppose that $\alpha \in \mathcal{S}$. We say $\alpha$ is vertical along $\mathcal{G}\left(\nu^{+}, \nu^{-}\right)$if its vertical length $v_{q}(\alpha)=i\left(\alpha, \nu^{-}\right)$vanishes, i.e., if $\alpha$ can be realized as a union of leaves of the vertical foliation. Similarly, $\alpha$ is horizontal if $h_{q}(\alpha)=i\left(\alpha, \nu^{+}\right)=0$. Mostly we shall be dealing with curves $\alpha$ which are neither horizontal nor vertical. In this case, there is always a unique time $t_{\alpha}$ at which $i\left(\alpha, \nu_{t_{\alpha}}^{+}\right)=i\left(\alpha, \nu_{t_{\alpha}}^{-}\right)$. This is when the $q$-length of $\alpha$ is shortest along $\mathcal{G}$. We call $t_{\alpha}$ the balance time of $\alpha$. Our estimation of the hyperbolic lengths of short curves will mainly be made relative to their balance time.

\section{Comparison on the thick part of Teichmüller space}

In this section we prove Theorem 3.9, which states that if $\mathcal{G}_{t}$ and $\mathcal{L}_{t}$ are in the $\epsilon_{0}$-thick part $\mathcal{T}_{\text {thick }}(S)$ of Teichmüller space, then the Teichmüller distance between them is uniformly bounded by a constant that depends only on the topology of $S$ and $\epsilon_{0}$. The idea is to first approximate the length of a curve $\zeta$ for any $\sigma \in \mathcal{T}_{\text {thick }}(S)$ by its intersection with what we call a short marking for $\sigma$, and then to compare the short markings for $\mathcal{G}_{t}$ and $\mathcal{L}_{t}$. This method will be extended in Section 7 when we consider the Teichmüller distance between $\mathcal{G}_{t}$ and $\mathcal{L}_{t}$ in general. We begin with some definitions.

3.1. Short markings. We call a maximal collection of pairwise disjoint, homotopically distinct, non-peripheral simple closed curves on $S$, a pants curve system on $S$. The terminology is due to the fact that the complementary components are pairs of pants, i.e., three holed spheres (in which some boundary components may be punctures). Our notion of a marking is motivated by [16]:

Definition 3.1. A marking $M$ on a surface $S$ is a system of pants curves $\alpha_{1}, \ldots, \alpha_{k}$ and simple closed curves $\delta_{\alpha_{1}}, \ldots, \delta_{\alpha_{k}}$ such that 
(i) $i\left(\alpha_{i}, \delta_{\alpha_{j}}\right)=0$ if $i \neq j$

(ii) $i\left(\alpha_{i}, \delta_{\alpha_{i}}\right)=2$ if two distinct pair of pants are adjacent along $\alpha_{i}$, $i\left(\alpha_{i}, \delta_{\alpha_{i}}\right)=1$ if $\alpha_{i}$ is adjacent to only a single pair of pants.

We call $\delta_{\alpha_{i}}$ the dual curve of $\alpha_{i}$.

In the first case of (ii), $\alpha_{i} \cup \delta_{\alpha_{i}}$ fill a four-holed sphere and in the second case $\alpha_{i} \cup \delta_{\alpha_{i}}$ fill a one-holed torus. It is easy to see that any two markings which have the same pants system $\mathcal{P}$ have dual curves which differ only by twists and half-twists around the curves in $\mathcal{P}$.

The following well-known lemma states that for any hyperbolic metric, one can always choose a pants system whose length is universally bounded:

Lemma 3.2. (Bers [3]) There exists a constant $L>0$ such that for every $\sigma \in \mathcal{T}(S)$ there is a pants curve system $\mathcal{P}$ with the property that $l_{\sigma}(\alpha)<L$ for every $\alpha \in \mathcal{P}$.

If the boundary curves of a pair of pants have bounded length as in Bers's lemma, the geometry of a pair of pants satisfies the following (for a proof, see Appendix):

Lemma 3.3. Let $P$ be a totally geodesic pair of pants with boundary curves $\alpha_{1}, \alpha_{2}, \alpha_{3}$ of lengths $l\left(\alpha_{i}\right)<L$ for $i=1,2,3$. Then the common perpendicular of $\alpha_{i}, \alpha_{j}$ (where possibly $i=j$ ) has length $\log \left[1 / l\left(\alpha_{i}\right)\right]+$ $\log \left[1 / l\left(\alpha_{j}\right)\right] \pm O(1)$, where the bound on the error depends only on $L$.

We will say that a pants curve system as in Lemma 3.2 is short in $(S, \sigma)$. A short marking is a short pants system together with a dual system chosen so that each dual curve $\delta_{\alpha_{i}}$ is the shortest among all possible dual curves. Notice that not all curves in a short marking are necessarily short; if a pants curve is very short, then the corresponding dual curve will be very long. More precisely, we have the following easy consequence of Lemma 3.3:

Corollary 3.4. Let $M_{\sigma}$ be a short marking for $\sigma$ and let $\alpha, \delta_{\alpha} \in M_{\sigma}$ be a pants curve and its dual. Then

$$
l_{\sigma}\left(\delta_{\alpha}\right)=i\left(\delta_{\alpha}, \alpha\right) \cdot 2 \log \left[1 / l_{\sigma}(\alpha)\right] \pm O(1)
$$

The notion of a short marking is useful because for surfaces in the thick part of $\mathcal{T}(S)$, short markings coarsely determine the geometry. In particular, we have the following proposition. See for example [18] Lemma 4.7 or Proposition 7.7 below for a proof. If $M$ is a marking and $\xi \in \mathcal{M L}(S)$, we write $i(M, \xi)=\sum_{\gamma \in M} i(\gamma, \xi)$. 
Proposition 3.5. Let $M_{\sigma}$ be a short marking for $\sigma \in \mathcal{T}_{\text {thick }}(S)$. Then for any $\zeta \in \mathcal{S}$,

$$
l_{\sigma}(\zeta) \stackrel{*}{\asymp} i\left(M_{\sigma}, \zeta\right) .
$$

Since both length and intersection number scale linearly with weights of simple closed curves, we find:

Proposition 3.6. Let $M_{\sigma}$ be a short marking for $\sigma \in \mathcal{T}_{\text {thick }}(S)$. Then for any $\xi \in \mathcal{M L}(S)$,

$$
l_{\sigma}(\xi) \stackrel{*}{=} i\left(M_{\sigma}, \xi\right) .
$$

3.2. Comparison on the thick part. We use the estimate in Proposition 3.6 to compare $\mathcal{G}_{t}$ and $\mathcal{L}_{t}$ in the thick part of $\mathcal{T}(S)$. The following two lemmas are well known, for convenience we furnish a proof. Recall that $\Sigma$ denotes the conformal structure associated to the metric $\sigma$.

Lemma 3.7. Suppose that $\sigma \in \mathcal{T}_{\text {thick }}(S)$ and $q \in \mathcal{Q}(\Sigma)$. Then for every $\zeta \in \mathcal{S}$

$$
l_{\sigma}(\zeta) \stackrel{*}{\leftrightharpoons} l_{q}(\zeta) .
$$

Proof. Let $F(S)$ be the bundle of unit quadratic differentials over $\mathcal{T}(S)$ whose fiber over a point $\Sigma$ is $\mathcal{Q}(\Sigma)$.

If the result were false, then there would exist a sequences of points $\sigma_{n} \in \mathcal{T}_{\text {thick }}(S)$, corresponding to Riemann surfaces $\Sigma_{n}$, of curves $\gamma_{n} \in \mathcal{S}$ and of quadratic differentials $q_{n} \in \mathcal{Q}\left(\Sigma_{n}\right)$, such that the ratio

$$
l_{\sigma_{n}}\left(\gamma_{n}\right) / l_{q_{n}}\left(\gamma_{n}\right)
$$

tends to zero or infinity. Since the projection of $\mathcal{T}_{\text {thick }}(S)$ to moduli space is compact, we can translate by elements in the mapping class group so that $\left\{\left(q_{n}, \Sigma_{n}\right)\right\} \subset F(S)$ lie over a compact set in $\mathcal{T}(S)$. Since by definition $q_{n}$ has unit norm, $\left\{\left(q_{n}, \Sigma_{n}\right)\right\}$ is contained in a compact subset of $F(S)$.

Renaming $\left(q_{n}, \Sigma_{n}\right)$, passing to a subsequence, and taking limits we may assume that $\Sigma_{n} \rightarrow \Sigma_{\infty} \in \mathcal{T}_{\text {thick }}(S)$ and that $q_{n} \rightarrow q_{\infty} \in \mathcal{Q}\left(\Sigma_{\infty}\right)$. This gives a sequence $\gamma_{n} \in \mathcal{S}$ for which $l_{\sigma_{\infty}}\left(\gamma_{n}\right) / l_{q_{\infty}}\left(\gamma_{n}\right)$ is unbounded above or below as $n \rightarrow \infty$, where $\sigma_{\infty}$ is the hyperbolic structure uniformizing $\Sigma_{\infty}$. Extracting a subsequence from $\gamma_{n}$ we may find constants $c_{n}$ such that $c_{n} \gamma_{n} \rightarrow \xi \in \mathcal{M L}(S)$. Since both $l_{\sigma_{\infty}}(\xi)$ and $l_{q_{\infty}}(\xi)$ are finite and positive, this is impossible.

Essentially the same proof shows that:

Lemma 3.8. For $\sigma \in \mathcal{T}(S)$ and $\gamma \in \mathcal{S}$, let $E_{\sigma}(\gamma)$ be the extremal length of $\gamma$ in $\sigma$. Then $l_{\sigma}^{2}(\gamma) \stackrel{*}{\asymp} E_{\sigma}(\gamma)$ for every $\gamma \in \mathcal{S}$. 
Proof. The method is the same as the above, on noting that $E_{\sigma}(c \gamma)=$ $c^{2} E_{\sigma}(\gamma)$.

Theorem 3.9. If $\mathcal{G}_{t}, \mathcal{L}_{t} \in \mathcal{T}_{\text {thick }}(S)$ then $d_{\mathcal{T}(S)}\left(\mathcal{G}_{t}, \mathcal{L}_{t}\right)=O(1)$.

Proof. By Lemma 3.7, Equation(2), and Proposition 3.6, we have

$$
\begin{aligned}
l_{g_{t}}\left(M_{g_{t}}\right) & \stackrel{*}{\asymp} l_{q_{t}}\left(M_{g_{t}}\right) \stackrel{*}{=} i\left(M_{g_{t}}, \nu_{t}^{+}\right)+i\left(M_{g_{t}}, \nu_{t}^{-}\right) \\
& \stackrel{*}{\asymp} l_{g_{t}}\left(\nu_{t}^{+}\right)+l_{g_{t}}\left(\nu_{t}^{-}\right) .
\end{aligned}
$$

Now, since $\mathcal{L}_{t}$ minimizes $l_{\sigma}\left(\nu_{t}^{+}\right)+l_{\sigma}\left(\nu_{t}^{-}\right)$over all $\sigma \in \mathcal{T}(S)$, we have

$$
l_{g_{t}}\left(\nu_{t}^{+}\right)+l_{g_{t}}\left(\nu_{t}^{-}\right)>l_{\mathcal{L}_{t}}\left(\nu_{t}^{+}\right)+l_{\mathcal{L}_{t}}\left(\nu_{t}^{-}\right) .
$$

Reversing the sequence of estimates in Equation(3), we get

$$
\begin{aligned}
l_{\mathcal{L}_{t}}\left(\nu_{t}^{+}\right)+l_{\mathcal{L}_{t}}\left(\nu_{t}^{-}\right) & \stackrel{*}{\asymp} i\left(M_{\mathcal{L}_{t}}, \nu_{t}^{+}\right)+i\left(M_{\mathcal{L}_{t}}, \nu_{t}^{-}\right) \stackrel{*}{`} l_{q_{t}}\left(M_{\mathcal{L}_{t}}\right) \\
& \stackrel{*}{=} l_{g_{t}}\left(M_{\mathcal{L}_{t}}\right) .
\end{aligned}
$$

Putting together the preceding three equations, we have

$$
l_{g_{t}}\left(M_{g_{t}}\right) \stackrel{*}{\succ} l_{g_{t}}\left(M_{\mathcal{L}_{t}}\right) .
$$

Since $g_{t} \in \mathcal{T}_{\text {thick }}(S)$ and since $M_{g_{t}}$ is a short marking, Corollary 3.4 implies that $l_{g_{t}}\left(M_{g_{t}}\right) \stackrel{*}{=} 1$ and so $l_{g_{t}}\left(M_{\mathcal{L}_{t}}\right) \stackrel{*}{ }$. .

By Proposition 3.5, for any curve $\zeta \in \mathcal{S}$, we have $l_{g_{t}}(\zeta) \stackrel{*}{\asymp} i\left(M_{g_{t}}, \zeta\right)$ and $l_{\mathcal{L}_{t}}(\zeta) \stackrel{*}{\asymp} i\left(M_{\mathcal{L}_{t}}, \zeta\right)$. Notice that the proof of Proposition 3.5 depends only on the fact that the lengths of all the curves in the marking $M_{\sigma}$ are uniformly bounded above. Since $l_{g_{t}}\left(M_{\mathcal{L}_{t}}\right) \stackrel{*}{=} 1$, it follows also that $l_{g_{t}}(\zeta) \stackrel{*}{`} i\left(M_{\mathcal{L}_{t}}, \zeta\right)$. Hence $l_{g_{t}}(\zeta) \stackrel{*}{=} l_{\mathcal{L}_{t}}(\zeta)$ for any $\zeta \in$ $\mathcal{S}$. We deduce from Lemma 3.8 and Kerckhoff's formula $d_{\mathcal{T}}(\sigma, \tau)=$ $1 / 2 \sup _{\zeta \in \mathcal{S}} E_{\sigma}(\zeta) / E_{\tau}(\zeta)$ for Teichmüller distance [9], that $d_{\mathcal{T}}(\sigma, \tau)=$ $O(1)$.

\section{Twists And Fenchel Nielsen COORdinates}

In order to compare surfaces in the thin part of Teichmüller space, our main tool will be Minsky's product region theorem [19]. This uses Fenchel Nielsen coordinates to give a nice coarse expression for Teichmüller distance between surfaces which have common thin parts. To state the results precisely, we first discuss twists and Fenchel Nielsen coordinates. 
4.1. Twists in hyperbolic metrics. There are various ways to define the twist of one curve around another, all of which differ by factors unimportant to us here. We shall follow Minsky [19]. Let $\sigma \in \mathcal{T}(S)$ be a hyperbolic metric and let $\alpha$ be an oriented simple closed geodesic on $(S, \sigma)$. Let $\zeta$ be a simple geodesic that intersects $\alpha$ and let $p$ be a point of intersection. In the universal cover $\mathbb{H}^{2}$, a lift $\tilde{\zeta}$ intersects $\tilde{\alpha}$ at a lift $\tilde{p}$ of $p$, and has endpoints $\zeta_{R}, \zeta_{L}$ on $\partial_{\infty} \mathbb{H}^{2}$ to the right and left of $\alpha$, respectively (see Figure 1 ). Let $p_{R}, p_{L}$ be the orthogonal projections

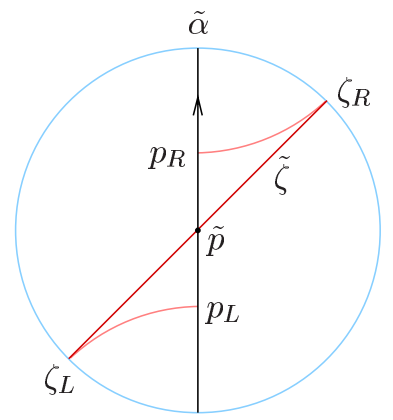

Figure 1. Defining the twist of $\zeta$ around $\alpha$.

of $\zeta_{R}, \zeta_{L}$ to $\tilde{\alpha}$ respectively. Then the twist of $\zeta$ around $\alpha$ at $p$ is defined as

$$
t w_{\sigma}(\zeta, \alpha, p)= \pm \frac{d_{\mathbb{H}^{2}}\left(p_{R}, p_{L}\right)}{l_{\sigma}(\alpha)},
$$

where the sign is $(+)$ if the direction from $p_{L}$ to $p_{R}$ coincides with the orientation of $\tilde{\alpha}$ and $(-)$ if it is opposite. For any other point $q \in \zeta \cap \alpha$, the twist satisfies $\left|t w_{\sigma}(\zeta, \alpha, q)-t w_{\sigma}(\zeta, \alpha, p)\right| \leq 1$ ([19] Lemma 3.1). To obtain a number that is independent of the point of intersection, Minsky defines

$$
t w_{\sigma}(\zeta, \alpha)=\min _{p \in \zeta \cap \alpha} t w_{\sigma}(\zeta, \alpha, p) .
$$

For convenience, we write $T w_{\sigma}(\zeta, \alpha)$ for $\left|t w_{\sigma}(\zeta, \alpha)\right|$.

We see that we can just as well define the twist of a geodesic lamination around $\alpha$. We remark that although we will be working with measured geodesic laminations, when defining the twist, the measure is irrelevant, in other words the twist $t w_{\sigma}(\nu, \alpha)$ depends only on the underlying lamination $|\nu|$.

4.2. Fenchel Nielsen coordinates. We define the Fenchel Nielsen coordinates

$$
\left(l_{\sigma}\left(\alpha_{i}\right), s_{\alpha_{i}}(\sigma)\right)_{i=1}^{k}
$$


associated to a pants curves system $\alpha_{1}, \ldots, \alpha_{k}$ in the following standard way, see for example [19]. First, construct a base surface $\sigma_{0}=$ $\sigma_{0}\left(l_{1}^{0}, \ldots, l_{k}^{0}\right)$ in which the pants curve $\alpha_{i}$ has some specific choice of length $l_{i}^{0}$, and in which all three "seams", i.e., the common perpendiculars between distinct pants curves in each pair of pants, match up with the seams in any adjacent pair of pants. Let $\gamma_{i}$ denote the curves formed by gluing the seams in this way. Any other structure $\sigma \in \mathcal{T}(S)$ comes endowed with an associated homeomorphism $h: \sigma_{0} \rightarrow \sigma$. The length coordinates of $\sigma$ are defined by $l_{\sigma}\left(\alpha_{i}\right)$. Let $\sigma_{0}\left(l_{1}, \ldots, l_{k}\right)$ denote the surface in which $\alpha_{i}$ (more precisely $h\left(\alpha_{i}\right)$ ) has length $l_{i}=l_{\sigma}\left(\alpha_{i}\right)$, while the curves formed by gluing the common perpendiculars are exactly the images $h\left(\gamma_{i}\right)$. Now define $\widehat{s}_{\alpha_{i}}(\sigma)$ to be the signed distance that one has to twist around $\alpha_{i}$ to obtain $\sigma$ starting from $\sigma_{0}\left(l_{1}, \ldots, l_{k}\right)$, where the sign is determined relative to a fixed orientation on $\sigma_{0}$ and hence on $\sigma$. Finally we define the twist coordinates of $\sigma$ by $s_{\alpha_{i}}(\sigma)=\widehat{s}_{\alpha_{i}}(\sigma) / l_{\sigma}\left(\alpha_{i}\right) \in \mathbb{R}$.

Lemma 4.1. (Minsky [19] Lemma 3.5) For any lamination $\nu \in \mathcal{M L}(S)$ and any two metrics $\sigma, \sigma^{\prime} \in \mathcal{T}(S)$,

$$
\left|\left(t w_{\sigma}(\nu, \alpha)-t w_{\sigma^{\prime}}(\nu, \alpha)\right)-\left(s_{\alpha}(\sigma)-s_{\alpha}\left(\sigma^{\prime}\right)\right)\right| \leq 4 .
$$

In [19], the statement is only given for closed curves. However the argument extends without change to laminations, the key step being the topological observation that for any two simple closed curves $\zeta_{1}, \zeta_{2}$ intersecting $\alpha$, the difference $t w_{\sigma}\left(\zeta_{1}, \alpha\right)-t w_{\sigma}\left(\zeta_{2}, \alpha\right)$ is a topological quantity, independent of $\sigma$, up to a bounded error of 1 . This extends to leaves of geodesic laminations crossing $\alpha$, since we can transfer lifts of specific leaves from one metric to another using the natural boundary map between limit sets, see for example [8] Section 3.7.

4.3. Relative twist in an annulus. The above topological observation allows us to define the algebraic intersection of two curves in an annulus:

Definition 4.2. For any two laminations $\nu_{1}, \nu_{2}$ that intersect a curve $\alpha$, define their algebraic intersection around $\alpha$ to be

$$
i_{\alpha}\left(\nu_{1}, \nu_{2}\right)=\inf _{\sigma}\left[t w_{\sigma}\left(\nu_{1}, \alpha\right)-t w_{\sigma}\left(\nu_{2}, \alpha\right)\right],
$$

where the infimum is taken over all possible surfaces $\sigma \in \mathcal{T}(S)$.

Often we need only the absolute value:

Definition 4.3. For any two laminations $\nu_{1}, \nu_{2}$ that intersect a curve $\alpha$, define the relative twisting of $\nu_{1}, \nu_{2}$ around $\alpha$ to be

$$
d_{\alpha}\left(\nu_{1}, \nu_{2}\right)=\left|i_{\alpha}\left(\nu_{1}, \nu_{2}\right)\right| \text {. }
$$


Thus for any $\sigma \in \mathcal{T}(S)$, we have $\left|t w_{\sigma}\left(\nu_{1}, \alpha\right)-t w_{\sigma}\left(\nu_{2}, \alpha\right)\right|=d_{\alpha}\left(\nu_{1}, \nu_{2}\right)+$ $O(1)$. Notice that $i_{\alpha}\left(\nu_{1}, \nu_{2}\right)$ and $d_{\alpha}\left(\nu_{1}, \nu_{2}\right)$ are independent of the measures on $\nu_{1}, \nu_{2}$, depending only on the underlying laminations $\left|\nu_{1}\right|$ and $\left|\nu_{2}\right|$.

Using Definition 4.2, one sees easily that

$$
i_{\alpha}\left(\nu_{1}, \nu_{2}\right)=i_{\alpha}\left(\nu_{1}, \xi\right)-i_{\alpha}\left(\nu_{2}, \xi\right)+O(1)
$$

for any curve $\xi$ transverse to $\alpha$. It is also easily seen that $d_{\alpha}\left(\nu_{1}, \nu_{2}\right)$ agrees up to $O(1)$ with the definition of subsurface distance between the projections of $\left|\nu_{1}\right|$ and $\left|\nu_{2}\right|$ to the annular cover of $S$ with core $\alpha$, as defined in [16] Section 2.4 and used throughout [20, 21].

Another essentially equivalent way of measuring twist is to look at the intersection with the shortest curve transverse to $\alpha$ :

Lemma 4.4. Let $\alpha$ be a pants curve and let $\delta_{\alpha}$ be the shortest dual curve of $\alpha$ in some marking for $\sigma$. Then for any simple closed curve $\zeta$ intersecting $\alpha$, we have $\left|t w_{\sigma}(\zeta, \alpha)-i_{\alpha}\left(\zeta, \delta_{\alpha}\right)\right|=O(1)$.

Proof. Since $i_{\alpha}\left(\zeta, \delta_{\alpha}\right)=t w_{\sigma}(\zeta, \alpha)-t w_{\sigma}\left(\delta_{\alpha}, \alpha\right)$, up to a bounded error of 1 , it is sufficient to show that $\left|t w_{\sigma}\left(\delta_{\alpha}, \alpha\right)\right|=O(1)$.

Let $\tilde{\delta}_{\alpha}$ be a lift of $\delta_{\alpha}$ in the universal cover $\mathbb{H}^{2}$ and let $\tilde{\alpha}, \tilde{\alpha}^{\prime}$ be the two lifts of $\alpha$ containing the endpoints of $\tilde{\delta}_{\alpha}$ (see Figure 2). Let $\eta$ be the perpendicular between $\tilde{\alpha}$ and $\tilde{\alpha}^{\prime}$ and let $p, p^{\prime}$ be the endpoints of $\eta$

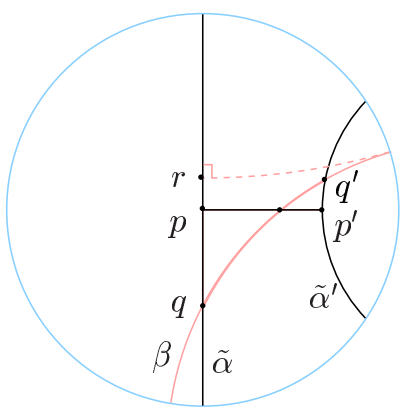

Figure 2. Bound on the twist of $\delta_{\alpha}$ around $\alpha$.

on $\tilde{\alpha}, \tilde{\alpha}^{\prime}$, respectively. Since $\delta_{\alpha}$ is the shortest dual curve, the endpoints of $\tilde{\delta}_{\alpha}$ must be within distance $l_{\sigma}(\alpha)$ from $p, p^{\prime}$. Let $q, q^{\prime}$ be points on $\tilde{\alpha}, \tilde{\alpha}^{\prime}$ at distance $l_{\sigma}(\alpha)$ from $p, p^{\prime}$, respectively, on opposite sides of $\eta$. It is easy to see that $\left|t w_{\sigma}\left(\delta_{\alpha}, \alpha\right)\right| \leq\left|t w_{\sigma}(\beta, \alpha)\right|$, where $\beta$ is the geodesic through $q, q^{\prime}$. Let $r$ be the foot of the perpendicular as shown. As in the first part of the proof of Lemma 3.5 in [19], we note that since the images of $\tilde{\alpha}^{\prime}$ under the translation along $\tilde{\alpha}$ are disjoint, the projection 
of $\tilde{\alpha}^{\prime}$ on $\tilde{\alpha}$ has length at most $l_{\sigma}(\alpha)$. Thus $l(p r)<l(p q)=l(\alpha)$. Hence, $\left|t w_{\sigma}(\beta, \alpha)\right|=2[l(p q)+l(p r)] / l(\alpha)<4$.

4.4. The product region theorem. Let $\mathcal{A} \subset \mathcal{S}$ be a collection of disjoint, homotopically distinct, simple closed curves on $S$ and let $\mathcal{T}_{\text {thin }}\left(\mathcal{A}, \epsilon_{0}\right) \subset \mathcal{T}(S)$ be the subset on which all curves $\alpha \in \mathcal{A}$ have hyperbolic length at most $\epsilon_{0}$. Extend $\mathcal{A}$ to a pants decomposition and define Fenchel Nielsen coordinates as above. Let $S_{\mathcal{A}}$ denote the surface obtained from $S$ by removing all the curves in $\mathcal{A}$ and replacing the resulting boundary components by punctures.

Following [19], we now define a projection $\Pi: \mathcal{T}(S) \rightarrow \mathcal{T}\left(S_{\mathcal{A}}\right) \times$ $\mathbb{H}_{\alpha_{1}} \times \ldots \times \mathbb{H}_{\alpha_{r}}$, where $\mathcal{A}=\left\{\alpha_{1} \ldots, \alpha_{r}\right\}$ and $\mathbb{H}_{\alpha_{i}}$ is the upper-half plane. The first component $\Pi_{0}$ which maps to $\mathcal{T}\left(S_{\mathcal{A}}\right)$ is defined by forgetting the coordinates of the curves in $\mathcal{A}$ and keeping the same Fenchel Nielsen coordinates for the remaining surface. For $\alpha \in \mathcal{A}$ define $\Pi_{\alpha}: \mathcal{T}(S) \rightarrow \mathbb{H}_{\alpha}$ by $\Pi_{\alpha}(\sigma)=s_{\alpha}(\sigma)+i / l_{\sigma}(\alpha) \in \mathbb{H}_{\alpha}$. Let $d_{\mathbb{H}_{\alpha}}$ be half the usual hyperbolic metric on $\mathbb{H}_{\alpha}$. Minsky's product region theorem states that, up to bounded additive error, Teichmüller distance on $\mathcal{T}_{\text {thin }}\left(\mathcal{A}, \epsilon_{0}\right)$ is equal to the sup metric on $\mathcal{T}\left(S_{\mathcal{A}}\right) \times \mathbb{H}_{\alpha_{1}} \times \ldots \times \mathbb{H}_{\alpha_{r}}$ :

Theorem 4.5 (Minsky [19]). Let $\sigma, \tau \in \mathcal{T}_{\text {thin }}\left(\mathcal{A}, \epsilon_{0}\right)$. Then

$$
d_{\mathcal{T}(S)}(\sigma, \tau)=\max _{\alpha \in \mathcal{A}}\left\{d_{\mathcal{T}\left(S_{\mathcal{A}}\right)}\left(\Pi_{0}(\sigma), \Pi_{0}(\tau)\right), d_{\mathbb{H}_{\alpha}}\left(\Pi_{\alpha}(\sigma), \Pi_{\alpha}(\tau)\right)\right\} \pm O(1) .
$$

We remark that Minsky makes several assumptions on the size of $\epsilon_{0}$ in order to prove the above theorem. We may assume that our initial choice of $\epsilon_{0}$ satisfies these assumptions. Recall from Section 5.3 that a curve $\alpha \in \mathcal{S}$ is said to be extremely short if $l_{\sigma}(\alpha)<\epsilon_{0}$.

The distance between the projections to $\mathbb{H}_{\alpha}$ can be approximated as follows:

Lemma 4.6. Suppose $\alpha \in \mathcal{S}$ is extremely short in both $\sigma, \tau$. Then

$$
\exp 2 d_{\mathbb{H}_{\alpha}}\left(\Pi_{\alpha}(\sigma), \Pi_{\alpha}(\tau)\right) \stackrel{*}{ } \max \left\{l_{\sigma} l_{\tau}\left|s_{\alpha}(\sigma)-s_{\alpha}(\tau)\right|^{2}, l_{\tau} / l_{\sigma}, l_{\sigma} / l_{\tau}\right\}
$$

where $l_{\sigma}=l_{\sigma}(\alpha)$ and $l_{\tau}=l_{\tau}(\alpha)$.

Proof. This is a simple calculation using the formula

$$
\cosh 2 d_{\mathbb{H}}\left(z_{1}, z_{2}\right)=1+\frac{\left|z_{1}-z_{2}\right|^{2}}{2 \operatorname{Im} z_{1} \operatorname{Im} z_{2}}
$$

where $d_{\mathbb{H}}$ is as above half the usual hyperbolic distance in $\mathbb{H}$.

Corollary 4.7. Suppose that $\sigma_{1}, \sigma_{2} \in \mathcal{T}_{\text {thin }}\left(\alpha, \epsilon_{0}\right)$ and $\nu \in \mathcal{M L}(S)$ satisfy $T w_{\sigma_{i}}(\nu, \alpha) l_{\alpha}\left(\sigma_{i}\right)=O(1)$ for $i=1,2$. Then

$$
d_{\mathbb{H}_{\alpha}}\left(\Pi_{\alpha}\left(\sigma_{1}\right), \Pi_{\alpha}\left(\sigma_{2}\right)\right)=\left|\log \left[l_{\sigma_{1}}(\alpha) / l_{\sigma_{2}}(\alpha)\right]\right| \pm O(1) .
$$


Proof. This follows easily from Lemma 4.6, using Lemma 4.1 to approximate $s_{\alpha}\left(\sigma_{1}\right)-s_{\alpha}\left(\sigma_{2}\right)$ by $t w_{\sigma_{1}}(\nu, \alpha)-t w_{\sigma_{2}}(\nu, \alpha)$. (Cross terms such as $s_{\alpha}\left(\sigma_{1}\right) l_{\sigma_{2}}(\alpha)$ may be rearranged as $\left[s_{\alpha}\left(\sigma_{1}\right) l_{\sigma_{1}}(\alpha)\right]\left[l_{\sigma_{2}}(\alpha) / l_{\sigma_{1}}(\alpha)\right]$.) Note that the multiplicative error in Lemma 4.6 translates to an additive error in the distance.

\section{Short Curves Along Teichmüller GeOdesics}

In this section we prove Theorem A, stated more precisely as Theorem 5.8. This gives a combinatorial estimate for the hyperbolic length of an extremely short curve along the Teichmüller geodesic $\mathcal{G}\left(\nu^{+}, \nu^{-}\right)$. We also recall the estimate for the twist of $\nu^{ \pm}$around such curves (Theorem 5.10) proved in [21].

Deriving the length estimate is largely a matter of putting together results proved in $[17,20,22]$. Both estimates are made by relating the hyperbolic metric $g_{t}$ and the quadratic differential metric $q_{t}$. As indicated in the discussion in the introduction following Theorem A, there are two distinct reasons why a curve $\alpha$ may become extremely short; one is that the relative twisting of $\nu^{+}$and $\nu^{-}$around $\alpha$ may be very large, while the other relates to a scale factor which controls the relationship between the quadratic differential and hyperbolic metrics on the components of the thick part of $g_{t}$ adjacent to $\alpha$. For this we need Rafi's thick-thin decomposition for quadratic differentials, Theorem 5.5. We begin by reviewing some essential facts about the geometry of annuli with respect to quadratic differential metrics.

5.1. Annuli in quadratic differential metrics. In this section, we review the notion of a flat annulus and expanding annulus defined in [19]. These concepts provide the framework with which to analyze short curves.

Let $\Sigma \in \mathcal{T}(S)$ and let $q$ be a quadratic differential on $\Sigma$. Let $\gamma$ be a piecewise smooth curve in $(S, q)$. At a smooth point $p$, the curvature $\kappa(p)$ is well-defined, up to a choice of sign. If $\gamma$ is the boundary component of a subsurface $Y$, we choose the sign to be positive if the acceleration vector at $p$ points into $Y$. At a singular point $P$, although the curvature is not defined, we shall say $\gamma$ is non-negatively curved at $P$ if the interior angle $\theta(P)$ is at most $\pi$ and say it is non-positively curved at $P$ if $\theta(P)$ is at least $\pi$. We say $\gamma$ is monotonically curved with respect to $Y$ either if the curvature is non-negative at every point, or non-positive at every point. The total curvature of $\gamma$ is given by

$$
\kappa_{Y}(\gamma)=\int_{\gamma} \kappa(p)+\sum[\pi-\theta(P)]
$$


where the sum is taken over all singular points $P$ on $\gamma$. The GaussBonnet theorem gives

$$
\sum_{i} \kappa_{Y}\left(\gamma_{i}\right)-\pi \sum_{j} \operatorname{ord} P_{j}=2 \pi \chi(Y)
$$

where $\operatorname{ord} P_{j}$ is the order of the zero at $P_{j}$, the first sum is over all boundary components $\gamma_{i}$ of $Y$, and the second sum is over the zeros $P_{j}$ of $q$ in the interior of $Y$.

Let $A$ be an annulus in $(S, q)$ with piecewise smooth boundary. The following definitions are due to Minsky [17]. We say $A$ is regular if both boundary components $\partial_{0}, \partial_{1}$ are monotonically curved with respect to $A$ and if $\partial_{0}, \partial_{1}$ are $q$-equidistant from each other. Suppose that $A$ is a regular annulus such that $\kappa\left(\partial_{0}\right) \leq 0$. We say $A$ is an expanding annulus if $\kappa\left(\partial_{0}\right)<0$ and we call $\partial_{0}$ the inner boundary and $\partial_{1}$ the outer boundary. Expanding annuli are exemplified by an annulus bounded by a pair of concentric circles in $\mathbb{R}^{2}$. The inner boundary is the circle of smaller radius and has curvature $-2 \pi$, while the outer boundary has curvature $2 \pi$.

A regular annulus is primitive if it contains no singularities in its interior. By Equation(5), its boundaries satisfy $\kappa\left(\partial_{0}\right)=-\kappa\left(\partial_{1}\right)$. A regular annulus is flat if $\kappa\left(\partial_{0}\right)=\kappa\left(\partial_{1}\right)=0$. By (5), a flat annulus is necessarily primitive, and is foliated by Euclidean geodesics homotopic to the boundaries. Thus a flat annulus is isometric to a cylinder obtained as the quotient of a Euclidean rectangle in $\mathbb{R}^{2}$. Note that a primitive annulus must either be flat or expanding.

5.2. Modulus of annulus and length of short curve. Let $\sigma$ be the hyperbolic metric that uniformizes $\Sigma$. If $\alpha$ is short in $\sigma$, Maskit [13] showed that the extremal length $E_{\Sigma}(\alpha)$ and hyperbolic length $l_{\sigma}(\alpha)$ are comparable, up to multiplicative constants. Moreover, there is an embedded collar $C(\alpha)$ around $\alpha$ in $(S, \sigma)$ whose modulus is comparable to $1 / l_{\sigma}(\alpha)$ (see [19] for an explicit calculation), and therefore also to $1 / E_{\Sigma}(\alpha)$. By definition of extremal length, this implies that the maximal annulus around $\alpha$ in $\Sigma$ has modulus comparable to $1 / l_{\sigma}(\alpha)$. The following theorem of Minsky allows us to replace any annulus of sufficiently large modulus with one that is primitive:

Theorem 5.1. (17] Theorem 4.6) Let $A \subset S$ be any homotopically non-trivial annulus whose modulus is sufficiently large. Then $A$ contains a primitive annulus $B$ such that $\operatorname{Mod} A \asymp \operatorname{Mod} B$.

(The statement of Theorem 4.6 in [17] should $\operatorname{read} \operatorname{Mod} A \geq m_{0}$ not $\operatorname{Mod} A \leq m_{0}$.) Thus, we have 
Theorem 5.2. If $\alpha$ is a simple closed curve which is sufficiently short in $(S, \sigma)$, then for any $q \in \mathcal{Q}(\Sigma)$, there is a primitive annulus $A$ with core homotopic to $\alpha$ such that

$$
\frac{1}{l_{\sigma}(\alpha)} \asymp \operatorname{Mod}(A) .
$$

We may assume that $\epsilon_{0}$ was chosen so that if $l_{\sigma}(\alpha)<\epsilon_{0}$, then $l_{\sigma}(\alpha)$ is small enough that this theorem is valid.

The reason for introducing flat or expanding annuli is that their moduli are easy to estimate. The following result can be deduced from Theorem 4.5 of [17] and is proved in [20]:

Theorem 5.3. Let $A \subset S$ be a primitive annulus with inner and outer boundaries $\partial_{0}$ and $\partial_{1}$, respectively. Let $d$ be the q-distance between $\partial_{0}$ and $\partial_{1}$. Then either

(i) $A$ is flat and $\operatorname{Mod} A=d / l_{q}\left(\partial_{0}\right)$ or

(ii) $A$ is expanding and $\operatorname{Mod} A \asymp \log \left[d / l_{q}\left(\partial_{0}\right)\right]$.

We will apply this theorem in the following context. It follows from Equation(5) that every simple closed curve $\gamma$ on $(S, q)$ either has a unique $q$-geodesic representative, or is contained in a family of closed Euclidean geodesics which foliate a flat annulus [25]. Denote by $F(\gamma)$ the maximal flat annulus, which necessarily contains all $q$-geodesic representatives of $\gamma$. If the geodesic representative of $\gamma$ is unique, then $F(\gamma)$ is taken to be the degenerate annulus containing this geodesic alone. Denote the (possibly coincident) boundary curves of $F(\gamma)$ by $\partial_{0}, \partial_{1}$ and consider the $q$-equidistant curves from $\partial_{i}$ outside $F(\gamma)$. Let $\hat{\partial}_{i}$ denote the first such curve which is not embedded. The pair $\partial_{i}$, $\hat{\partial}_{i}$ bound a region $E_{i}(\gamma)$ whose interior is an annulus with core homotopic to $\gamma$, and which is by its construction regular and expanding. Notice that $l_{q}(\gamma)=l_{q}\left(\partial_{0}\right)=l_{q}\left(\partial_{1}\right)$ and that by [20] Lemma 3.6, $d_{q}\left(\partial_{i}, \hat{\partial}_{i}\right) \asymp l_{q}\left(\hat{\partial}_{i}\right)$ (where $d_{q}$ is the $q$-distance between the boundary components). From Theorems 5.1 and 5.2 we have:

Corollary 5.4. If $\alpha$ is an extremely short curve on $(S, \sigma)$, then

$$
1 / l_{\sigma}(\alpha) \asymp \max \left\{\operatorname{Mod} F(\alpha), \operatorname{Mod} E_{0}(\alpha), \operatorname{Mod} E_{1}(\alpha)\right\} .
$$

The idea of our basic length estimates for extremely short curves $\alpha$ in Theorem 5.8, is to combine this corollary with the estimates for the moduli of $F(\alpha)$ and $E_{i}(\alpha)$ in Theorem 5.3.

5.3. Thick-thin decomposition and the $q$-metric. The thick-thin decomposition for quadratic differentials developed in [22] describes the relationship between the $q$-metric on surface $\Sigma$ and the uniformizing 
hyperbolic metric $\sigma$ in the thick components of the thick-thin decomposition of $\sigma$. It states that on the hyperbolic thick parts of $(S, \sigma)$ the two metrics are comparable, up to a factor which depends on the moduli of the expanding annuli around the short curves in the boundary of the thick component. This factor will be crucial in our estimates below.

To make a precise statement, for a subsurface $Y$ of $S$, define the $q$-geodesic representative of $Y$ to be the unique subsurface $\hat{Y}$ of $(S, q)$ with $q$-geodesic boundary in the homotopy class of $Y$ that is disjoint from the interior of $F\left(\gamma_{i}\right)$ for all components $\gamma_{i}$ of $\partial Y$. Notice that $\hat{Y}$ is $q$-geodesically convex, so that if a closed curve $\zeta$ is contained in $Y$, it has a $q$-geodesic representative contained in $\hat{Y}$.

If $Y$ is not a pair of pants, define $\lambda_{Y}$ to be the length of the $q$ shortest non-peripheral simple closed curve contained in $\hat{Y}$. If $Y$ is a pair of pants, define $\lambda_{Y}$ to be $\max \left\{l_{q}\left(\gamma_{1}\right), l_{q}\left(\gamma_{2}\right), l_{q}\left(\gamma_{3}\right)\right\}$ where $\gamma_{1}, \gamma_{2}, \gamma_{3}$ are the three boundary curves of $\hat{Y}$. The thick-thin decomposition for quadratic differentials is the following:

Theorem 5.5 (Rafi [22]). Let $\sigma$ be the hyperbolic metric that uniformizes $\Sigma$ and let $Y$ be a thick component of the hyperbolic thick-thin decomposition of $(S, \sigma)$. Then

(i) $\operatorname{diam}_{q} \hat{Y} \stackrel{*}{`} \lambda_{Y}$,

(ii) For any non-peripheral simple closed curve $\zeta$ in $Y$, we have

$$
l_{q}(\zeta) \stackrel{*}{\asymp} \lambda_{Y} l_{\sigma}(\zeta)
$$

5.4. Length and twist along $\mathcal{G}\left(\nu^{+}, \nu^{-}\right)$. As explained at the end of Section 5.2, we can use Theorem 5.3 and Corollary 5.4 and to estimate the length of an extremely short curve $\alpha$ in $\mathcal{G}_{t} \in \mathcal{G}\left(\nu^{+}, \nu^{-}\right)$by calculating the modulus of the maximal flat annulus $F_{t}(\alpha)$ and the maximal expanding annuli $E_{t}(\alpha)$ around $\alpha$, for $E=E_{0}, E_{1}$.

Recall from Section 4.3 that $d_{\alpha}\left(\nu^{+}, \nu^{-}\right)$is the relative twisting of $\nu^{+}, \nu^{-}$around $\alpha$.

Proposition 5.6. The modulus of $F_{t}(\alpha)$ is given by

$$
\operatorname{Mod} F_{t}(\alpha) \asymp e^{-2\left|t-t_{\alpha}\right|} d_{\alpha}\left(\nu^{+}, \nu^{-}\right) .
$$

Proof. Since a flat annulus is Euclidean, its geometry is very simple. For a geodesic arc $\zeta$ transverse to $\alpha$, define $t w_{F_{t}}(\zeta, \alpha)$ to be the intersection number of $\zeta$ with a geodesic arc $\beta$ joining the two boundaries of $F_{t}(\alpha)$ and perpendicular to the $q$-representatives of $\alpha$. Assuming that $\alpha$ is neither vertical nor horizontal, then at the balance time $t_{\alpha}$ (see Section 2.8) the horizontal and vertical foliations both make an 
angle of $\pi / 4$ with the $q$-geodesic representatives of $\alpha$. In this case, the modulus of $F_{t_{\alpha}}(\alpha)$ is approximated by $t w_{F_{t_{\alpha}}}\left(\nu^{+}, \alpha\right)=t w_{F_{t_{\alpha}}}\left(\nu^{-}, \alpha\right)$. More generally, the horizontal leaves make an angle $\psi_{t}$ with $\alpha$, where $\left|\tan \psi_{t}\right|=e^{2\left(t-t_{\alpha}\right)}$. From this it is a straightforward exercise in Euclidean geometry, see [21], to prove:

$$
\left|t w_{F_{t}}\left(\nu^{ \pm}, \alpha\right)-e^{\mp 2\left(t-t_{\alpha}\right)} \operatorname{Mod} F_{t}(\alpha)\right| \leq 1 .
$$

We claim that

$$
\left|t w_{F_{t}}\left(\nu^{+}, \alpha\right)-t w_{F_{t}}\left(\nu^{-}, \alpha\right)\right|=d_{\alpha}\left(\nu^{+}, \nu^{-}\right)+O(1),
$$

from which the proposition follows. As in [21], define the twist $t w_{q_{t}}(\zeta, \alpha)$ of a geodesic arc $\zeta$ around $\alpha$, with respect to $q_{t}$ as follows. Let $\tilde{S}$ be the annular cover of $(S, q)$ and let $\tilde{\beta}$ be a bi-infinite geodesic arc in $\tilde{S}$ that is perpendicular to the lifts of the $q$-geodesic representatives of $\alpha$. Define $t w_{q_{t}}(\zeta, \alpha)$ to be the intersection number of $\tilde{\zeta}$ with $\tilde{\beta}$. A straightforward application of Equation(5) shows that twisting is essentially confined to the maximal flat annulus round $\alpha$ :

$$
t w_{q_{t}}(\zeta, \alpha)=t w_{F_{t}}(\zeta, \alpha)+O(1)
$$

It was observed in Section 4.2 (see also the subsequent discussion in Section 4.3) that although $t w_{\sigma}(\nu, \alpha)$ depends on the metric $\sigma$ in which it is measured, the difference in twist of $\nu^{+}$and $\nu^{-}$equals (up to a bounded error) the number of times a leaf of $\nu^{+}$intersects a leaf of $\nu^{-}$ in the annular cover of $S$ corresponding to $\alpha$. This is true even when the metric is a quadratic differential metric. Therefore,

$$
\begin{aligned}
\left|t w_{q}\left(\nu^{+}, \alpha\right)-t w_{q}\left(\nu^{-}, \alpha\right)\right| & =\left|t w_{\sigma}\left(\nu^{+}, \alpha\right)-t w_{\sigma}\left(\nu^{-}, \alpha\right)\right| \pm O(1) \\
& =d_{\alpha}\left(\nu^{+}, \nu^{-}\right) \pm O(1) .
\end{aligned}
$$

Equation(7) now follows from the preceding two equations.

We shall estimate the modulus of the expanding annuli using the following:

Proposition 5.7. Let $q \in \mathcal{Q}(\Sigma)$. Suppose that $\alpha$ is extremely short in $\sigma$ and let $Y$ be a thick component of the hyperbolic thick-thin decomposition of $(S, \sigma)$, one of whose boundary components is $\alpha$. Let $\hat{\alpha}$ be the q-geodesic representative of $\alpha$ on the boundary of $\hat{Y}$ and let $E(\alpha)$ be the maximal expanding annulus on the same side of $\hat{\alpha}$ as $\hat{Y}$. Then

$$
\operatorname{Mod} E(\alpha) \asymp \log \frac{\lambda_{Y}}{l_{q}(\alpha)} .
$$


Proof. Let $d_{q}$ denote the $q$-metric. By Theorem 5.3(ii), it is sufficient to show that $d_{q}\left(\partial_{0}, \partial_{1}\right) \asymp \lambda_{Y}$.

Note that although $E(\alpha)$ is not necessarily contained in $\hat{Y}$, the outer boundary $\partial_{1}$ must intersect $\hat{Y}$. Hence, $d_{q}\left(\partial_{0}, \partial_{1}\right) \leq \operatorname{diam}_{q}(\hat{Y})$ and so by Theorem $5.5(\mathrm{i})$, we have $d_{q}\left(\partial_{0}, \partial_{1}\right) \prec \lambda_{Y}$.

Now we prove the inequality in the other direction. Observe that since $E(\alpha)$ is maximal, the outer boundary $\partial_{1}$ intersects itself and so there is a non-trivial arc $\omega$ with endpoints on $\hat{\alpha}$ whose length is $2 d_{q}\left(\partial_{0}, \partial_{1}\right)$. First suppose that $\omega$ is contained in $\hat{Y}$. A regular neighborhood of $\alpha \cup \omega$ is a pair of pants whose boundary curves are $\alpha$ and two additional curves $\zeta_{1}, \zeta_{2}$. Note that for $i=1,2$,

$$
l_{q}(\omega)+l_{q}(\alpha) \geq l_{q}\left(\zeta_{i}\right) .
$$

Thus, if either $\zeta_{1}$ or $\zeta_{2}$, say $\zeta_{1}$ is non-peripheral in $Y$, then

$$
\frac{d_{q}\left(\partial_{0}, \partial_{1}\right)}{l_{q}(\alpha)} \succ \frac{l_{q}\left(\zeta_{1}\right)}{l_{q}(\alpha)} \geq \frac{\lambda_{Y}}{l_{q}(\alpha)} .
$$

If both $\zeta_{1}, \zeta_{2}$ are peripheral, then $Y$ is a pair of pants, and we have

$$
\frac{d_{q}\left(\partial_{0}, \partial_{1}\right)}{l_{q}(\alpha)} \succ \frac{l_{q}\left(\zeta_{1}\right)+l_{q}\left(\zeta_{2}\right)}{l_{q}(\alpha)} \asymp \frac{\lambda_{Y}}{l_{q}(\alpha)} .
$$

If $\omega$ exits $\hat{Y}$, we replace it with a new arc $\omega^{\prime}$ as follows. Let $p$ be the first exit point and let $\gamma$ be the boundary component of $\hat{Y}$ that contains $p$. Let $\omega^{\prime}$ be the arc that first goes along $\omega$ to $p$, then makes one turn around $\gamma$ from $p$ to itself, then comes back to $\alpha$ along the first path. Because $\gamma$ is in the boundary of $\hat{Y}$, its hyperbolic length is extremely short and thus by Theorem 5.2 , the original arc $\omega$ must pass through an annulus of large modulus with core curve $\gamma$. Therefore, $l_{q}(\gamma) \leq l_{q}(\omega)$ and so we have

$$
l_{q}\left(\omega^{\prime}\right) \leq 2 l_{q}(\omega)+l_{q}(\gamma) \leq 3 l_{q}(\omega) .
$$

Now we can run the same argument as above with $\omega^{\prime}$ in place of $\omega$ to deduce the desired inequality.

We are now able to write down the desired length estimate. Suppose that the curve $\alpha$ is extremely short in some surface $g_{t}$ along the Teichmüller geodesic $\mathcal{G}\left(\nu^{+}, \nu^{-}\right)$. Let $Y_{1}, Y_{2}$ be the thick components of the thick-thin decomposition of $\left(S, g_{t}\right)$ that are adjacent to $\alpha$ (where $Y_{1}$ may equal $Y_{2}$ ). Define

$$
K_{t}(\alpha)=\max \left\{\frac{\lambda_{Y_{1}}}{l_{q_{t}}(\alpha)}, \frac{\lambda_{Y_{2}}}{l_{q_{t}}(\alpha)}\right\}
$$


and

$$
D_{t}(\alpha)=e^{-2\left|t-t_{\alpha}\right|} d_{\alpha}\left(\nu^{+}, \nu^{-}\right)
$$

Then, combining Corollary 5.4 and Propositions 5.6 and 5.7, we obtain:

Theorem 5.8. Let $\alpha$ be a curve on $S$ that is neither vertical nor horizontal. If $\alpha$ is extremely short in $g_{t}$, then

$$
\frac{1}{l_{g_{t}}(\alpha)} \asymp \max \left\{D_{t}(\alpha), \log K_{t}(\alpha)\right\} .
$$

It was shown in [20] that $K_{t}(\alpha)$ can be formulated combinatorially as the subsurface intersection of $\nu^{+}, \nu^{-}$in the corresponding component of the thick part of $g_{t}$ adjacent $\alpha$. Since this is not necessary for our development, we shall not do this here.

We need to estimate not only the lengths but also the twist parameters about short curves. The following key result allows us to directly compare the twists in the hyperbolic and quadratic differential metrics.

Proposition 5.9 ([21] Theorem 4.3). Suppose that $\sigma$ is a hyperbolic metric uniformizing a surface $\Sigma \in \mathcal{T}(S)$ and that $q \in \mathcal{Q}(\Sigma)$ and let $\zeta$ be a geodesic arc transverse to $\alpha$. Then

$$
\left|t w_{\sigma}(\zeta, \alpha)-t w_{q}(\zeta, \alpha)\right|=O\left(1 / l_{\sigma}(\alpha)\right) .
$$

(The statement in [21] has $i_{\alpha}\left(\zeta, \delta_{\alpha}\right)$ in place of $t w_{\sigma}(\zeta, \alpha)$, however by Lemma 4.4, this distinction is unimportant.) Combining this with Equations(6),(8), Proposition 5.6, and Theorem 5.8, we get:

Theorem 5.10 ([21] Theorem 1.3). With the same hypotheses as Theorem 5.8 ,

$$
\begin{aligned}
& T w_{g_{t}}\left(\nu^{+}, \alpha\right)=O\left(\frac{1}{l_{g_{t}}(\alpha)}\right) \quad \text { if } t \geq t_{\alpha}, \\
& T w_{g_{t}}\left(\nu^{-}, \alpha\right)=O\left(\frac{1}{l_{g_{t}}(\alpha)}\right) \quad \text { if } t \leq t_{\alpha} .
\end{aligned}
$$

5.5. Estimates for horizontal and vertical short curves on $\mathcal{G}$. We also need the analogue of Theorems 5.8 and 5.10 for extremely short curves $\alpha$ which are either horizontal or vertical.

For definiteness, assume $\alpha$ is vertical so that $i\left(\alpha, \nu^{-}\right)=0$. The definition of balance time no longer makes sense. Instead, we work relative to time $t=0$. Let $d$ be the height (i.e., distance between the two boundaries) of $F_{0}(\alpha)$. At an arbitrary time $t, l_{q_{t}}(\alpha)=i\left(\alpha, \nu_{t}^{+}\right)=$ $e^{t} l_{q_{0}}(\alpha)$ while the height of $F_{t}(\alpha)$ is $e^{-t} d$. Hence

$$
\operatorname{Mod} F_{t}(\alpha)=e^{-2 t} \operatorname{Mod} F_{0}(\alpha) \text {. }
$$


This is the analogue of Proposition 5.6.

If $\alpha$ is vertical, the discussion in Proposition 5.7 about expanding annuli is unchanged. Thus we obtain:

Theorem 5.11. Let $\alpha$ be a vertical curve on $S$. If $\alpha$ is extremely short in $g_{t}$, then

$$
\frac{1}{l_{g_{t}}(\alpha)} \asymp \max \left\{e^{-2 t} \operatorname{Mod} F_{0}(\alpha), \log K_{t}(\alpha)\right\} .
$$

If $\alpha$ is horizontal, the estimate is the same except that the first term is replaced by $e^{2 t} \operatorname{Mod} F_{0}(\alpha)$.

We also want the analogue of Theorem 5.10. If $\alpha$ is vertical, then $T w_{g_{t}}\left(\nu^{-}, \alpha\right)$ is undefined. However we have:

Theorem 5.12. With the same hypotheses as Theorem 5.11, if $\alpha$ is vertical then

$$
T w_{g_{t}}\left(\nu^{+}, \alpha\right)=O\left(\frac{1}{l_{g_{t}}(\alpha)}\right)
$$

while if $\alpha$ is horizontal then

$$
T w_{g_{t}}\left(\nu^{-}, \alpha\right)=O\left(\frac{1}{l_{g_{t}}(\alpha)}\right) \text {. }
$$

Proof. If $\alpha$ is vertical, the $q$-twist $t w_{q}\left(\nu^{+}, \alpha\right)$ in $F_{t}(\alpha)$ vanishes, while if $\alpha$ is horizontal, then $t w_{q}\left(\nu^{-}, \alpha\right)=0$. The result follows from $\operatorname{Equation}(8)$ and Proposition 5.9.

\section{Short CURVES AlONG Lines of Minima}

In this section we prove Theorem B, stated more precisely as Theorem 6.1, which gives our our combinatorial estimate for the length of a curve which becomes extremely short at some point along the line of minima $\mathcal{L}\left(\nu^{+}, \nu^{-}\right)$. We also estimate the twist of $\nu^{ \pm}$around $\alpha$ in Theorem 6.2. This will form the basis for our comparison of the metrics $\mathcal{L}_{t}$ and $g_{t}$. It turns out that, in a close parallel to the case of the Teichmüller geodesic, there are two reasons why a curve can be extremely short: either the relative twisting of $\nu^{+}, \nu^{-}$about $\alpha$ is large, or one or other of the pants curves in a pair of pants adjacent to $\alpha$ in a short marking of $\mathcal{L}_{t}$ has large intersection with either $\nu^{+}$or $\nu^{-}$.

More precisely, suppose that $\alpha$ is an extremely short curve in $\mathcal{L}_{t}$ and let $\mathcal{P}_{\mathcal{L}_{t}}$ be a short pants system in $\mathcal{L}_{t}$, which necessarily contains $\alpha$. Define

$$
H_{t}(\alpha)=\sup _{\beta \in \mathcal{B}} \frac{l_{q_{t}}(\beta)}{l_{q_{t}}(\alpha)},
$$


where $\mathcal{B}$ is the set of pants curves in $\mathcal{P}_{\mathcal{L}_{t}}$ which are boundaries of pants adjacent to $\alpha$.

Let $D_{t}(\alpha)$ be as in Equation(10). Our main estimates are:

Theorem 6.1. Let $\alpha$ be a curve on $S$ which is neither vertical nor horizontal. If $\alpha$ is extremely short in $\mathcal{L}_{t}$, then

$$
\frac{1}{l_{\mathcal{L}_{t}}(\alpha)} \asymp \max \left\{D_{t}(\alpha), \sqrt{H_{t}(\alpha)}\right\} .
$$

Theorem 6.2. With the same hypotheses as Theorem 6.1, the twist satisfies:

$$
\begin{aligned}
& T w_{\mathcal{L}_{t}}\left(\nu^{+}, \alpha\right)=O\left(\frac{1}{l_{\mathcal{L}_{t}}(\alpha)}\right) \quad \text { if } t \geq t_{\alpha}, \\
& T w_{\mathcal{L}_{t}}\left(\nu^{-}, \alpha\right)=O\left(\frac{1}{l_{\mathcal{L}_{t}}(\alpha)}\right) \quad \text { if } t \leq t_{\alpha} .
\end{aligned}
$$

To prove Theorems $6.1,6.2$, we note that since the surface $\mathcal{L}_{t}$ is on the line of minima, we have at the point $\mathcal{L}_{t}$,

$$
\mathrm{d} l\left(\nu_{t}^{+}\right)+\mathrm{d} l\left(\nu_{t}^{-}\right)=0 .
$$

Theorems 6.1 and 6.2 will follow from the relations we get by applying Equation(13) to $\frac{\partial}{\partial \tau_{\alpha}}, \frac{\partial}{\partial l(\alpha)}$, which are respectively the infinitesimal twist and length deformations of $\alpha$. For the first of these we use the wellknown formula of Kerckhoff [10] and Wolpert [26], while for the second we use the analogous formula for the length deformation derived in [23].

6.1. Differentiation with respect to twist. Suppose as above that $\alpha$ is an extremely short curve in $\mathcal{L}_{t}$. If we apply Equation(13) to $\frac{\partial}{\partial \tau_{\alpha}}$, the derivative formula in [10] and [26] gives

$$
0=\frac{\partial l\left(\nu_{t}^{+}\right)}{\partial \tau_{\alpha}}+\frac{\partial l\left(\nu_{t}^{-}\right)}{\partial \tau_{\alpha}}=\int_{\alpha} \cos \theta^{+} d \nu_{t}^{+}+\int_{\alpha} \cos \theta^{-} d \nu_{t}^{-},
$$

where $\theta^{ \pm}$is the function measuring the angle from each arc of $\left|\nu_{t}^{ \pm}\right|$to $\alpha$. Assume that $\alpha$ is neither vertical nor horizontal, so that neither $i\left(\nu^{+}, \alpha\right)$ nor $i\left(\nu^{-}, \alpha\right)$ is zero. Then we may define the average angle $\Theta_{t}^{ \pm}$ by

$$
\cos \Theta_{t}^{ \pm}=\frac{1}{i\left(\nu_{t}^{ \pm}, \alpha\right)} \int_{\alpha} \cos \theta^{ \pm} d \nu_{t}^{ \pm}
$$

Setting $T=t-t_{\alpha}$, the preceding two equations give

$$
e^{T} \cos \Theta_{t}^{+}+e^{-T} \cos \Theta_{t}^{-}=0 \text {. }
$$


If a particular leaf $L$ of a lamination $|\nu|$ cuts $\alpha$ at an angle $\theta$ at a point $p$, then from the definition of the twist (see Section 4.1) and simple hyperbolic geometry we have

$$
\cos \theta=\tanh [t w(L, \alpha, p) l(\alpha) / 2] .
$$

Since the twists $t w(L, \alpha, p)$ for different leaves $L$ differ by at most 1 , if $\alpha$ is sufficiently short we obtain the estimate

$$
\left|\cos \theta-\cos \Theta_{t}^{ \pm}\right|=O(l(\alpha))
$$

from which we deduce that either $\cos \Theta_{t}^{ \pm}$and $t w_{\mathcal{L}_{t}}\left(\nu^{ \pm}, \alpha\right)$ have the same sign, or that $\left|\cos \Theta_{t}^{ \pm}\right|=O(l(\alpha))$ so that $\left|t w_{\mathcal{L}_{t}}\left(\nu^{ \pm}, \alpha\right)\right|=O(1)$.

Note also that Equation(14) implies that $\nu^{+}, \nu^{-}$twist around $\alpha$ in opposite directions and that the lamination whose weight on $\alpha$ is smaller does more of the twisting.

6.2. Differentiation with respect to length. For the length deformation, we shall apply the extension of the Wolpert formula derived in [23], which gives a general expression for $\mathrm{d} l(\zeta)$ with reference to a marking $M$, where $\zeta \in \mathcal{S}$. Let $\tilde{\alpha}_{1}, \ldots, \tilde{\alpha}_{n}$ be the lifts of the pants curves in $M$ successively met by $\zeta$, where the segment of the lift $\tilde{\zeta}$ of $\zeta$ between $\tilde{\alpha}_{1}$ and $\tilde{\alpha}_{n}$ projects to one complete period of $\zeta$. Let $d_{j}$ be the length of the common perpendicular $\pi_{j}$ between $\tilde{\alpha}_{j}, \tilde{\alpha}_{j+1}$ and let $S_{j}$ be the signed distance between $\pi_{j-1}$ and $\pi_{j}$ along $\tilde{\alpha}_{j}$, where the sign is positive if the direction from $\pi_{j-1}$ to $\pi_{j}$ coincides with the orientation of $\alpha_{j}$. Then Equation(3) of [23] states that

$$
\mathrm{d} l(\zeta)=\sum_{j=1}^{n} \cosh u_{j} \mathrm{~d} d_{j}+\sum_{j=1}^{n} \cos \theta_{j} \mathrm{~d} S_{j},
$$

where $\theta_{j}$ is the angle from $\tilde{\zeta}$ to $\tilde{\alpha}_{j}$ measured counter-clockwise and $u_{j}$ is the complex distance from $\tilde{\zeta}$ to $\pi_{j}$. Replacing sums by integrals, we see that this formula, derived in [23] for closed curves, pertains equally to a measured lamination.

We want to apply this formula to $\frac{\partial}{\partial l(\alpha)}$ for some specific curve $\alpha$, where, as in [23], we will take the partial derivative to mean with respect to a fixed set of coordinates $\left(l_{\sigma}(\alpha), \hat{s}_{\alpha}(\sigma)\right)$ for $\mathcal{T}(S)$, the twist coordinate $\hat{s}_{\alpha}(\sigma)$ being defined as in Section 4.2. The non-zero contributions will be from terms $\mathrm{d} S_{j}$ corresponding to lifts of $\alpha$, and from two types of term $\mathrm{d} d_{j}$ : those corresponding to perpendiculars with endpoints on lifts of $\alpha$, and those corresponding to perpendiculars which do not intersect any lift of $\alpha$, but whose projections are contained in a common pair of pants with $\alpha$. 
We first estimate the contribution from the terms $\mathrm{d} d_{j}$. Suppose as above that $\mathcal{P}_{\mathcal{L}_{t}}$ is a short pants decomposition for $\mathcal{L}_{t}$. Let $P$ be a pair of pants in $S \backslash \mathcal{P}_{\mathcal{L}_{t}}$ that has $\alpha$ as a boundary component. The geometry of $P$ is completely determined by the lengths of the three boundary curves $\alpha, \beta, \gamma$. A common perpendicular joining two (not necessarily distinct) boundary components of $P$ may or may not have one of its endpoints on a boundary curve which projects to $\alpha$ on $S$. We say that the common perpendiculars of the first kind are adjacent to $\alpha$, while those of the second type are not. The terms $\mathrm{d} d d_{j}$ are estimated by the following lemma which is proved in the Appendix:

Lemma 6.3. Let $P$ be a pair of pants in $S \backslash \mathcal{P}_{\mathcal{L}_{t}}$ that has $\alpha$ as a boundary component, and suppose that $\alpha$ is extremely short in $\mathcal{L}_{t}$. Let $u$ be the length of a common perpendicular adjacent to $\alpha$, and let $v$ denote the length of a common perpendicular not adjacent to $\alpha$. Then

$$
\begin{aligned}
& \text { (i) } \frac{\partial u}{\partial l(\alpha)} \stackrel{*}{=}-\frac{1}{l(\alpha)} \\
& \text { (ii) } \frac{\partial v}{\partial l(\alpha)} \stackrel{*}{=} l(\alpha),
\end{aligned}
$$

where the partial derivatives are taken with respect to Fenchel Nielsen coordinates defined using the pants decomposition $\mathcal{P}_{\mathcal{L}_{t}}$.

We remark that the first of these estimates coincides with the heuristic computation that since the collar around $\alpha$ has length comparable to $\log [1 / l(\alpha)]$, the derivative should be approximately $-1 / l(\alpha)$.

We also need to bound the coefficient $\cosh u_{j}$ of $\mathrm{d} d_{j}$ in Equation (16). Notice that the bound applies to all the pants curves (not just the extremely short ones) in a short marking.

Lemma 6.4. If $\alpha_{j}$ is a pants curve in a short marking, then

$$
\left|\cosh u_{j}\right| \stackrel{*}{ } 1 \text {. }
$$

Proof. Since $M$ is a short marking, by definition all curves $\alpha_{j}$ have length bounded above, and hence the length $d_{j}$ of the common perpendicular $\pi_{j}$ to $\tilde{\alpha}_{i}, \tilde{\alpha}_{j+1}$ is bounded below.

Suppose first that $\tilde{\zeta}$ intersects $\pi_{j}$ in a point $o$ whose perpendicular distance from $\tilde{\alpha}_{j}$ along $\pi_{j}$ is $x_{j}$. In this case, $u_{j}=i \phi_{j}$, where $\phi_{j}$ is the angle between $\tilde{\zeta}$ and $\pi_{j}$ at $o$. Since $\tilde{\zeta}$ intersects both $\tilde{\alpha}_{j}$ and $\tilde{\alpha}_{j+1}$, the angle of parallelism formula gives $\tan \phi_{j}<1 / \sinh x_{j}$ and $\tan \phi_{j}<1 / \sinh \left(d_{j}-x_{j}\right)$. Since at least one of $x_{j}$ and $d_{j}-x_{j}$ is bounded below, this gives a uniform upper bound on $\tan \phi_{j}$. Thus $\phi_{j}$ is bounded away from $\pi / 2$ and $\left|\cosh u_{j}\right|=\left|\cos \phi_{j}\right|$ is bounded away from 0 . 
If $\tilde{\zeta}$ is disjoint from $\pi_{j}$, then $u_{j}$ is the hyperbolic distance from $\tilde{\zeta}$ to $\pi_{j}$. Let $p$ denote the point where the common perpendicular from $\tilde{\zeta}$ to $\pi_{j}$ meets $\pi_{j}$. If $y_{j}$ denotes the distance from $p$ to $\tilde{\alpha}_{j}$, then the quadrilateral formula gives $\sinh y_{j} \sinh u_{j}=\left|\cos \theta_{j}\right|$, where $\theta_{j}$ is the angle between $\tilde{\zeta}$ and $\tilde{\alpha}_{j}$. Arguing as above, we may suppose that $y_{j}$ is bounded below, whence $\left|\sinh u_{j}\right|$ and hence $\left|\cosh u_{j}\right|$ are bounded above. The result follows.

We now consider the second sum in Equation(16).

Lemma 6.5. Let $\tilde{\alpha}_{j}$ be a lift of the curve $\alpha$ with corresponding shift coordinate $S_{j}=S_{j}(\zeta)$. Then

$$
\left|\frac{\partial S_{j}}{\partial l(\alpha)}-t w_{\mathcal{L}_{t}}(\zeta, \alpha)\right|=O(1)
$$

Proof. Homotope the lift $\tilde{\zeta}$ to the piecewise geodesic path $\hat{\zeta}$ that runs along the successive lifts $\tilde{\alpha}_{i}$ and common perpendiculars to the $\tilde{\alpha}_{i}$. The projection of $\hat{\zeta}$ to $S$ is homotopic to $\zeta$. Then $S_{j}(\zeta)$ equals the signed distance that $\hat{\zeta}$ travels along $\tilde{\alpha}_{j}$. We can express $S_{j}(\zeta)=$ $n_{j}(\zeta) l(\alpha) / 2+e_{j}(\zeta)+\hat{s}_{j}(\alpha)$ where $n_{j}(\zeta) \in \mathbb{Z}$ is the (signed) number of seams $\hat{\zeta}$ intersects along $\tilde{\alpha}_{j}$, where $\hat{s}_{j}(\alpha)$ is the Fenchel Nielsen twist at $\mathcal{L}_{t}$ as defined in Section 4.2 , and $e_{j}(\zeta)$ is an error term that allows for the fact that in the base surface $\sigma_{0}$, the incoming and outgoing perpendiculars $\pi_{j}, \pi_{j+1}$ may be offset along $\alpha$ by an amount of absolute value at most $l_{\sigma}(\alpha)$ which depends only on the geometry of the two adjacent pants, see [23]. In particular $e_{j}(\zeta)$ can be non-zero only in the case where either $\pi_{j}$ or $\pi_{j+1}$ projects to a perpendicular from $\alpha$ to itself.

Since the partial derivatives are taken with respect to the coordinates $\left(l_{\sigma}(\alpha), \hat{s}_{\sigma}(\alpha)\right)$, the term $\partial \hat{s}_{j} / \partial l(\alpha)$ vanishes. To avoid an unpleasant calculation, we get rid of the term $e_{j}(\zeta)$ as follows. Modify $\hat{\zeta}$ to a path which still runs along the lifts of the pants curves and their common perpendiculars, but which never goes along a perpendicular from a lift of $\alpha$ to itself. Specifically, let $\pi_{j}$ be such a common perpendicular which projects to a pair of pants $P$ one of whose boundary components is $\alpha$. Let $\beta$ be one of the other boundary components and let $\eta$ be the perpendicular from $\alpha$ to $\beta$. The projection of $\pi_{j}$ to $P$ is homotopic, with fixed endpoints, to an arc which runs along $\alpha$, then along $\eta$, then along $\beta$, back along $\eta$, finally back to the final point on $\alpha$, see Figure 3 . Modify $\hat{\zeta}$ by replacing $\pi_{j}$ by the lift of this alternate path. Doing this in each instance gives a replacement for $\hat{\zeta}$, with respect to which one 


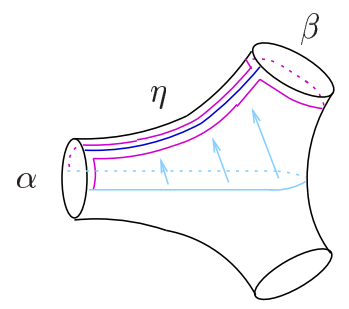

FiguRE 3. Homotoping the perpendicular.

can define all quantities occurring in (16) as before. The derivation of Equation (16) in [23] will still work for this new path. Denoting the newly defined shift also by $S_{j}$, we thus have $\partial S_{j} / \partial l(\alpha)=n_{j} / 2 \pm 1$. Now since $\delta_{\alpha}$ is the shortest dual curve to $\alpha$ at $\mathcal{L}_{t}$, it is not hard to see that $n_{j} / 2=i_{\alpha}\left(\zeta, \delta_{\alpha}\right) \pm O(1)$. The proof is completed by applying Lemma 4.4 .

We can now put the above results together to obtain an estimate of $\frac{\partial l(\nu)}{\partial l(\alpha)}$. Let $\left\{\pi_{j}\right\}_{j \in J}$ be the subset of perpendiculars whose projections are contained in a common pair of pants with $\alpha$ but are disjoint from $\alpha$. Then by Lemmas 6.3(ii) and 6.4, we have

$$
\sum_{j \in J} \cosh u_{j} \frac{\partial d_{j}}{\partial l(\alpha)} \stackrel{*}{`} \sum_{j \in J} \frac{\partial d_{j}}{\partial l(\alpha)} \stackrel{*}{`} \sum_{j \in J} l(\alpha) .
$$

In the case that we have a measured lamination $\nu$ instead of a curve $\zeta$, we obtain by the same reasoning

$$
\sum_{j \in J} \cosh u_{j} \frac{\partial d_{j}}{\partial l(\alpha)} \stackrel{*}{`} \sum_{j \in J} l(\alpha) \cdot w_{\nu}\left(\pi_{j}\right),
$$

where $w_{\nu}\left(\pi_{j}\right)$ is the $\nu$-weight of the leaves of $\tilde{\nu}$ that go from $\tilde{\alpha}_{j}$ to $\tilde{\alpha}_{j+1}$. Let us denote

$$
\Delta_{\nu}(\alpha)=\sum_{j \in J} w_{\nu}\left(\pi_{j}\right)
$$

By applying Equation(15), Lemmas 6.3 - 6.5 to Equation(16), we obtain the following:

Lemma 6.6. Let $\alpha$ be a curve in a short pants decomposition $\mathcal{P}$ of $\sigma$ and let $\nu$ be a measured lamination transverse to $\alpha$, with average intersection angle $\Theta$. If $\alpha$ is extremely short and neither horizontal nor vertical, then, using Fenchel Nielsen coordinates relative to $\mathcal{P}$, we 
have $\frac{\partial l(\nu)}{\partial l(\alpha)}=-A+B+C$, where

$A \triangleq i(\nu, \alpha) \frac{1}{l(\alpha)}, \quad B \stackrel{*}{ } \Delta_{\nu}(\alpha) l(\alpha), \quad C \stackrel{*}{\asymp} i(\nu, \alpha)(t w(\nu, \alpha) \cos \Theta+O(1))$.

6.3. Proof of the main estimates. We are ready to prove our main results Theorems 6.1 and 6.2. If we apply Equation(13) to $\partial / \partial l(\alpha)$, then by Lemma 6.6, we obtain

$$
0=\frac{\partial l\left(\nu_{t}^{+}\right)}{\partial l(\alpha)}+\frac{\partial l\left(\nu_{t}^{-}\right)}{\partial l(\alpha)}=-\left(A^{+}+A^{-}\right)+B^{+}+B^{-}+C^{+}+C^{-}
$$

where

$$
\begin{aligned}
& A^{ \pm} \stackrel{*}{\asymp} i\left(\nu_{t}^{ \pm}, \alpha\right) \frac{1}{l(\alpha)}, B^{ \pm} \stackrel{*}{\asymp} \Delta_{\nu_{t}^{ \pm}}(\alpha) l(\alpha) \\
& C^{ \pm} \stackrel{*}{\asymp} i\left(\nu_{t}^{ \pm}, \alpha\right)\left(t w\left(\nu^{ \pm}, \alpha\right) \cos \Theta_{t}^{ \pm}+O(1)\right) .
\end{aligned}
$$

Since $A^{+}+A^{-}=B^{+}+B^{-}+C^{+}+C^{-}$, we get

$$
\frac{1}{l(\alpha)} \stackrel{*}{\asymp} \frac{\Delta_{\nu_{t}^{+}}(\alpha)+\Delta_{\nu_{t}^{-}}(\alpha)}{i\left(\nu_{t}^{+}, \alpha\right)+i\left(\nu_{t}^{-}, \alpha\right)} l(\alpha)+\frac{C^{+}+C^{-}}{i\left(\nu_{t}^{+}, \alpha\right)+i\left(\nu_{t}^{-}, \alpha\right)} .
$$

Lemma 6.7. Let $H_{t}(\alpha)$ be defined as in Equation(12). Then we have

$$
H_{t}(\alpha) \asymp \frac{\Delta_{\nu_{t}^{+}}(\alpha)+\Delta_{\nu_{t}^{-}}(\alpha)}{i\left(\nu_{t}^{+}, \alpha\right)+i\left(\nu_{t}^{-}, \alpha\right)} .
$$

Proof. The strands of $\nu_{t}^{ \pm}$which intersect pants adjacent to $\alpha$ but which are disjoint from $\alpha$, must intersect one of the curves in $\mathcal{B}$. Hence, by definition of $\Delta_{\nu_{t}^{ \pm}}(\alpha)$, we have

$$
\frac{\Delta_{\nu_{t}^{+}}(\alpha)+\Delta_{\nu_{t}^{-}}(\alpha)}{i\left(\nu_{t}^{+}, \alpha\right)+i\left(\nu_{t}^{-}, \alpha\right)} \prec \sum_{\beta \in \mathcal{B}} \frac{l_{q_{t}}(\beta)}{l_{q_{t}}(\alpha)} \stackrel{*}{=} H_{t}(\alpha) .
$$

To prove the inequality in the other direction, let $\beta \in \mathcal{B}$ be the curve that realizes the maximum in the definition of $H_{t}(\alpha)$. For $\nu=\nu_{t}^{ \pm}$, let $\nu_{\beta \alpha}, \nu_{\beta \beta}, \nu_{\beta \gamma}$ be the collections of strands of $\nu$ that run between $\beta$ and $\alpha$, from $\beta$ to itself, and between $\beta$ and $\gamma$, respectively. (As usual, there are different possible configurations of strands in each pants, in particular $\nu_{\beta \beta}$ may be empty. The inequalities which follow are however valid in all cases.) Denote the $\nu$-weight of these by $w\left(\nu_{\beta \alpha}\right), w\left(\nu_{\beta \beta}\right)$, and $w\left(\nu_{\beta \gamma}\right)$, 
respectively. Then

$$
\begin{aligned}
H_{t}(\alpha) & =\frac{l_{q_{t}}(\beta)}{l_{q_{t}}(\alpha)} \stackrel{*}{\asymp} \sum_{I=+,-} \frac{w\left(\nu_{\beta \alpha}^{I}\right)+w\left(\nu_{\beta \beta}^{I}\right)+w\left(\nu_{\beta \gamma}^{I}\right)}{l_{q}(\alpha)} \\
& \prec \sum_{I=+,-} \frac{w\left(\nu_{\beta \beta}^{I}\right)+w\left(\nu_{\beta \gamma}^{I}\right)}{l_{q}(\alpha)} \prec \frac{\Delta_{\nu_{t}^{+}}(\alpha)+\Delta_{\nu_{t}^{-}}(\alpha)}{i\left(\nu_{t}^{+}, \alpha\right)+i\left(\nu_{t}^{-}, \alpha\right)} .
\end{aligned}
$$

Proof of Theorem 6.1. By Lemma 6.7, Equation(19) gives $1 / l_{\mathcal{L}_{t}}(\alpha) \asymp$ $G_{t}+H_{t} l_{\mathcal{L}_{t}}(\alpha)$, where $H_{t}=H_{t}(\alpha)$ and

$$
G_{t}=G_{t}(\alpha)=\frac{C^{+}+C^{-}}{i\left(\nu_{t}^{+}, \alpha\right)+i\left(\nu_{t}^{-}, \alpha\right)} .
$$

Hence, we must have either

$$
\frac{1}{l_{\mathcal{L}_{t}}(\alpha)} \asymp G_{t} \quad \text { or } \quad \frac{1}{l_{\mathcal{L}_{t}}(\alpha)} \asymp H_{t} l_{\mathcal{L}_{t}}(\alpha),
$$

from which we obtain

$$
\frac{1}{l_{\mathcal{L}_{t}}(\alpha)} \asymp \max \left\{G_{t}, \sqrt{H_{t}}\right\} .
$$

We simplify the expression for $G_{t}$ as follows. By the discussion following Equation(15) we see that either $t w_{\mathcal{L}_{t}}\left(\nu^{+}, \alpha\right) \cos \Theta_{t}^{+}$is positive, or $\left|t w_{\mathcal{L}_{t}}\left(\nu^{+}, \alpha\right) \cos \Theta_{t}^{+}\right|=O(1)$, and likewise for $\nu^{-}$. Also note that $t w_{\mathcal{L}_{t}}\left(\nu^{+}, \alpha\right)$ and $t w_{\mathcal{L}_{t}}\left(\nu^{-}, \alpha\right)$ are either $O(1)$ or have opposite signs, so that $d_{\alpha}\left(\nu^{+}, \nu^{-}\right)=T w_{\mathcal{L}_{t}}\left(\nu^{+}, \alpha\right)+T w_{\mathcal{L}_{t}}\left(\nu^{-}, \alpha\right) \pm O(1)$. As before, let $T=t-t_{\alpha}$. Then by applying Equation(14), we get

$$
\begin{aligned}
G_{t}(\alpha) & =\frac{e^{T}\left|\cos \Theta_{t}^{+}\right| T w_{\mathcal{L}_{t}}\left(\nu^{+}, \alpha\right)+e^{-T}\left|\cos \Theta_{t}^{-}\right| T w_{\mathcal{L}_{t}}\left(\nu^{-}, \alpha\right)}{e^{T}+e^{-T}}+O(1) \\
(20) & \asymp \frac{e^{T}\left|\cos \Theta_{t}^{+}\right| d_{\alpha}\left(\nu^{+}, \nu^{-}\right)}{e^{T}+e^{-T}}=\frac{e^{-T}\left|\cos \Theta_{t}^{-}\right| d_{\alpha}\left(\nu^{+}, \nu^{-}\right)}{e^{T}+e^{-T}} .
\end{aligned}
$$

This almost completes the proof, except it remains to be shown that if $1 / l_{\mathcal{L}_{t}}(\alpha) \asymp G_{t}$, then $G_{t} \asymp e^{-2\left|t-t_{\alpha}\right|} d_{\alpha}\left(\nu^{+}, \nu^{-}\right)$. By Equation(20), it is sufficient to show that there is some constant $c>0$, independent of $\alpha$, such that $\left|\cos \Theta_{t}^{-}\right|>c$ whenever $T>0$ and $\left|\cos \Theta_{t}^{+}\right|>c$ whenever $T<0$.

Our assumption that $1 / l_{\mathcal{L}_{t}}(\alpha) \asymp G_{t}$ and the fact that $l_{\mathcal{L}_{t}}(\alpha)$ is sufficiently small, together with Equation(20) imply that

$$
\frac{1}{l_{\mathcal{L}_{t}}(\alpha)} \stackrel{*}{\prec} T w_{\mathcal{L}_{t}}\left(\nu^{-}, \alpha\right)+T w_{\mathcal{L}_{t}}\left(\nu^{+}, \alpha\right) \text {. }
$$


Let $X_{t}=T w_{\mathcal{L}_{t}}\left(\nu^{-}, \alpha\right) l_{\mathcal{L}_{t}}(\alpha)$ and $Y_{t}=T w_{\mathcal{L}_{t}}\left(\nu^{+}, \alpha\right) l_{\mathcal{L}_{t}}(\alpha)$. The above inequality states that

$$
X_{t}+Y_{t} \stackrel{*}{\succ} 1 \text {. }
$$

If $T>0$ then by Equation(14), $\left|\cos \Theta_{t}^{-}\right|>\left|\cos \Theta_{t}^{+}\right|$so that $X_{t}>$ $Y_{t}-O(l(\alpha))$ by Equation(15). Thus, reducing the value of the upper bound $\epsilon_{0}$ on $l_{\mathcal{L}_{t}}(\alpha)$ if necessary, it follows from Equation(21) that $X_{t}$ is bounded below by some positive constant, and thus the same is true of $\left|\cos \Theta_{t}^{-}\right|$. The analogous statement holds for $\left|\cos \Theta_{t}^{+}\right|$when $T<0$.

Proof of Theorem 6.2. From Equation(14), $\left|\cos \Theta_{t}^{ \pm}\right| \leq e^{-2|T|}$. It follows from Equation(15) that if $T \gg 0$ then $T w_{\mathcal{L}_{t}}\left(\nu^{+}, \alpha\right) l_{\mathcal{L}_{t}}(\alpha) \prec e^{-2 T}$. The argument for $T \ll 0$ is similar.

Now suppose that $|T|=O(1)$. Since

$$
T w_{\mathcal{L}_{t}}\left(\nu^{ \pm}, \alpha\right) l_{\mathcal{L}_{t}}(\alpha) \prec d_{\alpha}\left(\nu^{+}, \nu^{-}\right) l_{\mathcal{L}_{t}}(\alpha)
$$

and since by Theorem $6.1, d_{\alpha}\left(\nu^{+}, \nu^{-}\right) l_{\mathcal{L}_{t}}(\alpha) \prec e^{2|T|}$, the result follows.

6.4. Estimates for horizontal and vertical short curves on $\mathcal{L}$. As in Section 5.5, we need the analogue of Theorems 6.1 and 6.2 for extremely short curves $\alpha$ which are either horizontal or vertical. As in that section, assume $\alpha$ is vertical so that $i\left(\alpha, \nu^{-}\right)=0$.

As before, we shall obtain the estimates by applying Equation(13) to $\frac{\partial}{\partial \tau_{\alpha}}, \frac{\partial}{\partial l(\alpha)}$. Since $\alpha$ is vertical,

$$
0=\frac{\partial l\left(\nu_{t}^{+}\right)}{\partial \tau_{\alpha}}+\frac{\partial l\left(\nu_{t}^{-}\right)}{\partial \tau_{\alpha}}=\frac{\partial l\left(\nu_{t}^{+}\right)}{\partial \tau_{\alpha}}=\int_{\alpha} \cos \theta^{+} d \nu_{t}^{+} .
$$

Hence Equation(14) is replaced by $\cos \Theta_{t}^{+}=0$. Furthermore, Equation(15) gives $\left|t w_{\mathcal{L}_{t}}\left(\nu^{+}, \alpha\right)\right|=O(1)$.

Let $m^{-}(\alpha)$ be the weight on $\alpha$ of $\nu^{-}=\nu_{0}^{-}$, in other words, $\nu^{-}=$ $m^{-}(\alpha) \alpha+\eta$, where $\eta$ has support disjoint from $\alpha$. Then following the line of discussion in Section 6.2, it is easy to check that

$$
\frac{\partial l\left(\nu_{t}^{-}\right)}{\partial l(\alpha)}=e^{-t} m^{-}(\alpha)+\Delta_{\nu_{t}^{-}}(\alpha) l(\alpha) .
$$

Hence, in place of Equation(18), we obtain

$$
0=\frac{\partial l\left(\nu_{t}^{+}\right)}{\partial l(\alpha)}+\frac{\partial l\left(\nu_{t}^{-}\right)}{\partial l(\alpha)}=-A^{+}+B^{+}+C^{+}+e^{-t} m^{-}(\alpha)+B^{-},
$$

where $A^{+}, B^{ \pm}, C^{+}$are defined as before. Since

$$
C^{+} \stackrel{*}{\asymp} i\left(\nu_{t}^{+}, \alpha\right)\left[t w\left(\nu^{+}, \alpha\right) \cos \Theta_{t}^{+}+O(1)\right] \stackrel{*}{\asymp} i\left(\nu_{t}^{+}, \alpha\right),
$$


we get

$$
\frac{1}{l_{\mathcal{L}_{t}}(\alpha)} \asymp H_{t}(\alpha) l_{\mathcal{L}_{t}}(\alpha)+\frac{e^{-t} m^{-}(\alpha)}{i\left(\nu_{t}^{+}, \alpha\right)}=H_{t}(\alpha) l_{\mathcal{L}_{t}}(\alpha)+e^{-2 t} \frac{m^{-}(\alpha)}{i\left(\nu^{+}, \alpha\right)} .
$$

Thus we obtain:

Theorem 6.8. Let $\alpha$ be a curve which is vertical on $S$. If $\alpha$ is extremely short in $\mathcal{L}_{t}$, then

$$
\frac{1}{l_{\mathcal{L}_{t}}(\alpha)} \asymp \max \left\{e^{-2 t} \frac{m^{-}(\alpha)}{i\left(\nu^{+}, \alpha\right)}, \sqrt{H_{t}(\alpha)}\right\} .
$$

If $\alpha$ is horizontal, the estimate is the same except that the first term is replaced by $e^{2 t} m^{+}(\alpha) / i\left(\nu^{-}, \alpha\right)$, where now $m^{+}(\alpha)$ is the weight on $\alpha$ of $\nu^{+}$.

Theorem 6.9. With the same hypotheses as Theorem 6.8, the twist satisfies $T w_{\mathcal{L}_{t}}\left(\nu^{+}, \alpha\right)=O(1)$ if $\alpha$ is vertical and $T w_{\mathcal{L}_{t}}\left(\nu^{-}, \alpha\right)=O(1)$ if $\alpha$ is horizontal.

\section{Comparing $\mathcal{L}_{t}$ AND $\mathcal{G}_{t}$}

In this section, we compare the geometry of $\mathcal{L}_{t}$ and $\mathcal{G}_{t}$ by comparing their respective thick-thin decompositions. Specifically, we prove that

$$
H_{t}(\alpha) \asymp K_{t}(\alpha)
$$

which, together with Theorems 5.8 and 6.1 gives a precise relationship between the lengths of short curves on $\mathcal{L}_{t}$ and $g_{t}$. Furthermore, we show in Theorem 7.10 that on corresponding thick components, the two metrics $\mathcal{L}_{t}$ and $g_{t}$ almost coincide. Combining this with the information of twist given in Theorems 5.10 and 6.2, we estimate the Teichmüller distance between $\mathcal{L}_{t}$ and $\mathcal{G}_{t}$ in Theorem 7.15.

As explained in the introduction, the actual logical flow in proving Equation(22) is not straightforward. We first show relatively easily in Proposition 7.1 that $H_{t}(\alpha) \succ K_{t}(\alpha)$. From this we deduce that a curve that is extremely short in $g_{t}$ is also extremely short in $\mathcal{L}_{t}$. This is used in proving Theorem 7.10 mentioned above, which in turn is used in proving $H_{t}(\alpha) \prec K_{t}(\alpha)$.

\subsection{Curves are shorter in $\mathcal{L}\left(\nu^{+}, \nu^{-}\right)$.}

Proposition 7.1. If $\alpha$ is extremely short in $g_{t}$, then $H_{t}(\alpha) \succ K_{t}(\alpha)$. Therefore,

$$
\frac{1}{l_{\mathcal{L}_{t}}(\alpha)} \succ \frac{1}{l_{g_{t}}(\alpha)}
$$


Proof. Once we show that $H_{t}(\alpha) \succ K_{t}(\alpha)$, the second statement follows from Theorems 5.8 and 6.1.

The only case of interest will be that in which $K_{t}(\alpha)$ is large. Let $E(\alpha)$ be the one of the expanding annuli $E_{i}(\alpha)$ around $\alpha$ of larger modulus, defined as in the discussion preceding Corollary 5.4. Denote the inner and outer boundary curves of $E(\alpha)$ by $\partial_{0}$ and $\partial_{1}$. Let $\omega$ be an essential arc from $\alpha$ to itself such that $l_{q}(\omega)=2 d_{q}\left(\partial_{0}, \partial_{1}\right)$, where as usual $d_{q}$ denotes distance in the $q$-metric. The annulus $E(\alpha)$ intersects the $q$-representative $\hat{Y}$ of a thick component $Y$ of $\left(S, g_{t}\right)$ adjacent to $\alpha$. Let us first suppose that $\omega$ is contained in $\hat{Y}$. A small regular neighborhood of $\alpha \cup \omega$ has boundary consisting of $\alpha$ and two curves, $\zeta_{1}, \zeta_{2}$, which together with $\alpha$ bound a pair of pants. Therefore, either both $\zeta_{1}$ and $\zeta_{2}$ are contained in $\mathcal{B}$ or one of these two curves must intersect a curve in $\mathcal{B}$ transversely. (As in Equation(12), $\mathcal{B}$ is the set of pants curves in a short pants decomposition $\mathcal{P}_{\mathcal{L}_{t}}$ which are boundaries of pants adjacent to $\alpha$.)

First consider the case when $\zeta_{1}, \zeta_{2} \in \mathcal{B}$. If either $\zeta_{1}$ or $\zeta_{2}$ is nonperipheral in $Y$, then by definition of $H_{t}(\alpha)$ and $\lambda_{Y}$,

$$
H_{t}(\alpha)=\max _{\beta \in \mathcal{B}}\left\{\frac{l_{q}(\beta)}{l_{q}(\alpha)}\right\} \geq \max \left\{\frac{l_{q}\left(\zeta_{1}\right)}{l_{q}(\alpha)}, \frac{l_{q}\left(\zeta_{2}\right)}{l_{q}(\alpha)}\right\} \geq \frac{\lambda_{Y}}{l_{q}(\alpha)} .
$$

If both $\zeta_{1}, \zeta_{2}$ are peripheral in $Y$, then

$$
H_{t}(\alpha) \succ \frac{l_{q}\left(\zeta_{1}\right)+l_{q}\left(\zeta_{2}\right)+l_{q}(\alpha)}{l_{q}(\alpha)} \asymp \frac{\lambda_{Y}}{l_{q}(\alpha)} .
$$

Now consider the case when either $\zeta_{1}$ or $\zeta_{2}$ intersects a curve $\beta \in \mathcal{B}$ transversely. Note that for $i=1,2$,

$$
l_{q}\left(\zeta_{i}\right) \leq l_{q}(\omega)+l_{q}(\alpha) \leq 4 \operatorname{diam}_{q}(\hat{Y}) \stackrel{*}{=} \lambda_{Y} .
$$

Suppose that $\beta$ intersects $\zeta_{i}$ and that $\zeta_{i}$ is not peripheral in $Y$. Then it follows from the above inequality and the definition of $\lambda_{Y}$ that $l_{q}\left(\zeta_{i}\right) \stackrel{*}{ }$ $\lambda_{Y}$. Since by Theorem 5.5 we have $l_{q}\left(\zeta_{i}\right) \stackrel{*}{\leftrightharpoons} \lambda_{Y} l_{g}\left(\zeta_{i}\right)$, we see that $l_{g}\left(\zeta_{i}\right) \stackrel{*}{\asymp} 1$. Then, by the collar lemma for quadratic differentials [20], we have

$$
l_{q}(\beta) \stackrel{*}{\succ} l_{q}\left(\zeta_{i}\right) \stackrel{*}{ } \lambda_{Y} .
$$

The only remaining possibility to consider is when both $\zeta_{1}, \zeta_{2}$ are peripheral so that $Y$ is a pair of pants. If $\beta$ intersects $\zeta_{i}$, then since $\beta$ must pass through an annulus around $\zeta_{i}$ which has large modulus, we conclude $l_{q}(\beta) \succ l_{q}\left(\zeta_{i}\right)$. If $\beta$ does not intersect $\zeta_{i}$, any arc $\eta$ of $\beta \cap \hat{Y}$ has endpoints on the other curve $\zeta_{j}, j \neq i$. The curve that goes along $\eta$, winds once around $\zeta_{j}$ and comes back to $\alpha$ along $\eta$ is homotopic $\zeta_{i}$. 
Since $\beta$ passes through an annulus of large modulus around $\zeta_{j}$, this implies $l_{q}(\beta) \stackrel{*}{\succ}^{*} 2 l_{q}(\eta)+l_{q}\left(\zeta_{j}\right) \geq l_{q}\left(\zeta_{i}\right)$. Either way, we have $l_{q}(\beta) \stackrel{*}{\succ}^{\prime} l_{q}\left(\zeta_{i}\right)$ and thus $l_{q}(\beta) \stackrel{*}{\succ} l_{q}\left(\zeta_{1}\right)+l_{q}\left(\zeta_{2}\right)$.

Finally, if the original arc $\omega$ was not contained in $\hat{Y}$, we can replace it with an arc that is contained in $\hat{Y}$ of comparable length as in the proof of Theorem 5.7, and run the same argument.

The key point in proving the other half of Equation(22) is Proposition 7.4 , which shows that the metric $\mathcal{L}_{t}$ not only minimizes $l_{\sigma}\left(\nu_{t}^{+}\right)+$ $l_{\sigma}\left(\nu_{t}^{-}\right)$, but that it also in a suitable coarse sense minimizes the contribution to the sum of the parts of $\nu_{t}^{ \pm}$which lie in the thick part of $\mathcal{L}_{t}$. We begin with estimates that are necessary to analyze this thick part.

7.2. Length estimates on subsurfaces. If $(S, \sigma)$ is a hyperbolic surface and $Y \subset S$ is a subsurface of the thick part, we want to find an approximation to $l_{\sigma}\left(\nu^{ \pm} \cap Y\right)$. To consider the problem in general, we consider $l_{\sigma}\left(\nu^{ \pm} \cap Q\right)$ for a subsurface $Q$ with geodesic boundary. Suppose that $\zeta$ is a geodesic that intersects $Q$. The essential idea is that we can approximate $\zeta \cap Q$ by piecewise geodesic arcs homotopic to $\zeta \cap Q$, which alternately run along arcs perpendicular to $\partial Q$ and parallel to $\partial Q$. The length of the parallel portion is determined by the twisting of $\zeta$ about the curves in $\partial Q$, while the portion $\zeta_{Q}$ perpendicular to $\partial Q$ is defined and estimated as explained below.

Let $\underline{\alpha}$ be a collection of disjoint simple closed geodesics on $(S, \sigma)$ and let $Q$ be a totally geodesic surface which is the metric completion of a component of $S \backslash \underline{\alpha}$. (It is possible for two distinct boundary components of $Q$ to be identified in $S$ to a single curve $\alpha \in \underline{\alpha}$, so strictly speaking, $Q$ is not a subsurface of $S$.) If $\eta$ is an essential arc with with endpoints on $\partial Q$, let $\eta_{Q}$ be the shortest arc in $Q$ that is freely homotopic to $\eta$, relative to $\partial Q$. In this case, clearly $\eta_{Q}$ is orthogonal to $\partial Q$. If $\eta$ is a simple closed curve in $Q$, let $\eta_{Q}=\eta$. For convenience we allow the possibility that $\eta=\eta_{Q}$ is a component of $\partial Q$, remarking that this is not quite the same as the definition in [19].

Suppose $\xi$ is a measured lamination on $S$. Then the intersection $\xi \cap Q$ is a union of closed curves in $Q$ and arcs with endpoints on $\partial Q$. For a component $\eta$ of $\xi \cap Q$, let $n\left(\eta_{Q}\right)$ denote the transverse measure of arcs or curves in the homotopy class $\left[\eta_{Q}\right]$. The orthogonal projection of $\xi$ onto $Q$ is $\xi_{Q}=\sum n\left(\eta_{Q}\right) \eta_{Q}$, where the sum is taken over the representative $\eta_{Q}$ from each class of components, and correspondingly let $l_{\sigma}\left(\xi_{Q}\right)=\sum n\left(\eta_{Q}\right) l_{\sigma}\left(\eta_{Q}\right)$.

If all curves in $\partial Q$ are of bounded length, then we have the following estimate of $l_{\sigma}(\zeta \cap Q)$ in terms of $\zeta_{Q}$ and $T w_{\sigma}(\zeta, \alpha)$ : 
Lemma 7.2. Suppose $l_{\sigma}\left(\alpha_{j}\right)<\ell$ for every component $\alpha_{j}$ of $\partial Q$. Then there exists a constant $K=K(\ell)$ such that for any measured lamination $\xi$ on $S$ :

$$
\left|l_{\sigma}(\xi \cap Q)-\left[l_{\sigma}\left(\xi_{Q}\right)+\sum l_{\sigma}\left(\alpha_{j}\right) \frac{T w_{\sigma}\left(\xi, \alpha_{j}\right)}{2} i\left(\xi, \alpha_{j}\right)\right]\right| \leq K \cdot i(\xi, \partial Q),
$$

where the sum is taken over all $\alpha_{j}$ that are not components of $\xi$.

For a proof, see the Appendix. The next lemma can be proved similarly, applying the same property of hyperbolic triangles. We omit the proof.

Lemma 7.3. Suppose $l_{\sigma}(\alpha)<\epsilon_{0}$. Let $A$ be an embedded annulus in $(S, \sigma)$ such that one component of $\partial A$ is the geodesic $\alpha$ and the other a hyperbolically equidistant curve of length $\epsilon_{0}$. Then there is a uniform constant $K$ such that for any measured lamination $\xi$ on $S$ that intersects a transversely:

$$
\left|l_{\sigma}(\xi \cap A)-\left[\log \frac{1}{l_{\sigma}(\alpha)}+l_{\sigma}(\alpha) \frac{T w_{\sigma}(\xi, \alpha)}{2}\right] i(\xi, \alpha)\right| \leq K \cdot i(\xi, \alpha) .
$$

Here, $\log \left[1 / l_{\sigma}(\alpha)\right]$ approximates the width of $A$, up to a bounded additive error.

We will now apply Lemmas 7.2 and 7.3 to prove Proposition 7.4 below, which in turn is the key step to proving Theorem 7.10.

For $\rho>0$ and a hyperbolic metric $\sigma \in \mathcal{T}(S)$, define

$$
\mathcal{S}_{\rho}(\sigma)=\left\{\alpha \in \mathcal{S}: l_{\sigma}(\alpha)<\rho\right\} .
$$

Proposition 7.1 implies that if $\alpha$ is extremely short in $g_{t}$, then we can choose a constant $\epsilon<\epsilon_{0}$, depending only on $\epsilon_{0}$, such that if $l_{g_{t}}(\alpha)<\epsilon$, then $l_{\mathcal{L}_{t}}(\alpha)<\epsilon_{0}$. In other words, we can choose $\epsilon<\epsilon_{0}$ so that

$$
\mathcal{S}_{\epsilon}\left(g_{t}\right) \subset \mathcal{S}_{\epsilon_{0}}\left(\mathcal{L}_{t}\right)
$$

Now, let $Q=Q_{t}$ be a component of $S \backslash \mathcal{S}_{\epsilon}\left(g_{t}\right)$. The metric $\mathcal{L}_{t}$ naturally endows $Q$ with the structure of hyperbolic surface with geodesic boundary, which by the above, satisfies $l_{\mathcal{L}_{t}}(\alpha)<\epsilon_{0}$ for all components $\alpha$ of $\partial Q$. Henceforth, fix a constant $c$ that satisfies $\epsilon_{0}<c<\epsilon_{\mathcal{M}}$. For $\sigma=g_{t}, \mathcal{L}_{t}$, or in general, any metric that makes $Q$ a hyperbolic surface with geodesic boundary components that are extremely short, define $C(\alpha, \sigma)$ to be the collar of $\alpha$ in $(Q, \sigma)$ such that one component of $\partial C(\alpha)$ is (the geodesic representative of) $\alpha$, and the other, the equidistant curve of length $c$. Because $c<\epsilon_{\mathcal{M}}$, the collars are all disjoint from 
one another. Let $\left(Q_{T}, \sigma\right)$ be the metric subsurface of $Q$ defined by:

$$
\left(Q_{T}, \sigma\right)=(Q, \sigma) \backslash \coprod_{\alpha \in \partial Q} C(\alpha, \sigma) .
$$

In particular, every component of $\partial Q_{T}$ has length $c$.

Since $\mathcal{L}_{t}$ is on the line of minima, we have $l_{\mathcal{L}_{t}}\left(\nu_{t}^{+}\right)+l_{\mathcal{L}_{t}}\left(\nu_{t}^{-}\right)<$ $l_{g_{t}}\left(\nu_{t}^{+}\right)+l_{g_{t}}\left(\nu_{t}^{-}\right)$. The contribution to this inequality from $Q_{T}$ is given as follows:

Proposition 7.4. If $Q$ is a component of $S \backslash \mathcal{S}_{\epsilon}\left(g_{t}\right)$, then

$$
l_{\mathcal{L}_{t}}\left(\nu^{+} \cap Q_{T}\right)+l_{\mathcal{L}_{t}}\left(\nu^{-} \cap Q_{T}\right) \stackrel{*}{\prec} l_{g_{t}}\left(\nu^{+} \cap Q_{T}\right)+l_{g_{t}}\left(\nu^{-} \cap Q_{T}\right) .
$$

To prove Proposition 7.4, we need the following two lemmas.

Lemma 7.5. Suppose $(Q, \sigma)$ is a hyperbolic surface with geodesic boundary such that $l_{\sigma}(\alpha)<\epsilon_{0}$ for all $\alpha \in \partial Q$. Then for any measured geodesic lamination $\xi$ on $(S, \sigma)$, we have

$$
l_{\sigma}\left(\xi \cap Q_{T}\right) \stackrel{*}{\leftrightharpoons} l_{\sigma}\left(\xi_{Q} \cap Q_{T}\right) .
$$

Proof. We consider $l_{\sigma}\left(\xi \cap Q_{T}\right)=l_{\sigma}(\xi \cap Q)-l_{\sigma}(\xi \cap \coprod C(\alpha))$ and apply Lemmas $7.2,7.3$ to the right-hand side. The proof is completed by observing that

$$
l_{\sigma}\left(\xi_{Q} \cap Q_{T}\right)=l_{\sigma}\left(\xi_{Q}\right)-\sum_{\alpha \in \partial Q} \log \frac{1}{l_{\sigma}(\alpha)} i(\xi, \alpha)+O(i(\xi, \partial Q))
$$

and that $l_{\sigma}\left(\xi \cap Q_{T}\right) \stackrel{\succ}{\succ}^{*} i\left(\xi, \partial Q_{T}\right)=i(\xi, \partial Q)$, due to the fact that every component of $\partial Q_{T}$ has an annular neighborhood of definite width.

Lemma 7.6. Suppose that $(Q, \sigma)$ and $\left(Q, \sigma^{\prime}\right)$ are two hyperbolic surfaces with geodesic boundary whose boundary components are all extremely short. Further assume that their Fenchel Nielsen coordinates with respect to some pants decomposition of $Q$ coincide, except possibly for the lengths and twists corresponding to components of $\partial Q$. Then for any simple closed curve or arc $\eta$ with endpoints on $Q$,

$$
l_{\sigma}\left(\eta_{Q} \cap Q_{T}\right) \stackrel{*}{=} l_{\sigma^{\prime}}\left(\eta_{Q} \cap Q_{T}\right) .
$$

Proof. This is essentially the same as a discussion in Minsky [19] page 283. The idea is that there is a $K$-bilipschitz homeomorphism $\left(Q_{T}, \sigma\right) \rightarrow$ $\left(Q_{T}, \sigma^{\prime}\right)$ with constant $K$ depending only on $\epsilon_{0}$. To see this, cut $Q$ into hexagons, all of which agree except those whose edges form part of $\partial Q$. Now truncate those hexagons which have an edge on $\partial Q$ by cutting off the collar round $\partial Q$ in such a way that the boundary of the truncated hexagon is the corresponding component of $\partial Q_{T}$. By our construction, 
the non-geodesic edges of the hexagons in the two surfaces are both equidistant curves of the same length $c$.

We define the required map piecewise from each possibly truncated hexagon in $\left(Q_{T}, \sigma\right)$ to the corresponding one in $\left(Q_{T}, \sigma^{\prime}\right)$. Since all the Fenchel Nielsen coordinates agree in the interior of $Q_{T}$, we only have to see that there is a bilipschitz map between the truncated parts of two hexagons with alternate sides $l_{1}, l_{2}, l_{3}$ and $l_{1}^{\prime}, l_{2}, l_{3}$, where $l_{1}, l_{1}^{\prime}<c$. Such a map is easy to construct and the result follows. Note also that $K \rightarrow 1$ as $\epsilon_{0} \rightarrow 0$.

We may assume that in the definition of the truncated surfaces $Q_{T}$, the constants $\epsilon_{0}$ and $c$ are chosen small enough that any non-peripheral geodesic loop contained in $(Q, \sigma)$ is completely contained in $Q_{T}$. In particular, if $\mathcal{P}_{\sigma}$ is a short pants system for $\sigma$ and if $\beta \in \mathcal{P}_{\sigma} \backslash \partial Q$ is contained in $Q_{T}$, then so is its dual $\delta_{\beta}$. Let $M_{\sigma}$ be the short marking of $\sigma$ associated to $\mathcal{P}_{\sigma}$. We call the subset of $M_{\sigma}$ thus defined, the restriction $\left.M_{\sigma}\right|_{Q}$ of $M_{\sigma}$ to $Q$ [16]. Equivalently, $\left.M_{\sigma}\right|_{Q}$ is the set of curves in $M_{\sigma}$ that are completely contained in $Q$ and are non-peripheral in $Q$. If $Q$ is a pair of pants then $\left.M_{\sigma}\right|_{Q}$ is empty.

Proof of Proposition 7.4. Where convenient, we drop the subscript $t$. Since $Q$ is a component of $S \backslash \mathcal{S}_{\epsilon}\left(g_{t}\right)$, the curves in $\partial Q$ are included in the set of pants curves in both $M_{\mathcal{L}}$ and $M_{g}$. Define a new metric $\tau=\tau_{t}$ on $S$ interpolating $g_{t}$ and $\mathcal{L}_{t}$ as follows. Let $X$ be the metric completion of $S \backslash Q$. First we choose a new pants system $\mathcal{P}_{\tau}$ for $S$. The system $\mathcal{P}_{\tau}$ contains all the curves in $\partial Q$, in the interior of $Q$ it consists of the pants curves in $\left.M_{g}\right|_{Q}$, while in the interior of $X$ it consists of the pants curves in $\left.M_{\mathcal{L}}\right|_{X}$. We define $\tau_{t}$ by specifying its Fenchel Nielsen coordinates with respect to $\mathcal{P}_{\tau}$. The metric $\tau_{t}$ will have the same Fenchel Nielsen coordinates associated to the pants curves in $\left.M_{g}\right|_{Q}$ as $g_{t}$ and the same Fenchel Nielsen coordinates associated to the curves in $\left.M_{\mathcal{L}}\right|_{Q} \cup \partial Q$ as $\mathcal{L}_{t}$.

Since $\mathcal{L}$ is on the line of minima we have:

$$
l_{\mathcal{L}}\left(\nu_{t}^{+}\right)+l_{\mathcal{L}}\left(\nu_{t}^{-}\right)<l_{\tau}\left(\nu_{t}^{+}\right)+l_{\tau}\left(\nu_{t}^{-}\right) .
$$

Let us estimate both sides of this inequality. Applying Lemma 7.2 to $\nu=\nu_{t}^{ \pm}$, we obtain:

$$
\begin{aligned}
& l_{\tau}(\nu \cap X)=l_{\tau}\left(\nu_{X}\right)+\frac{1}{2} \sum_{\alpha \in \partial Q} l_{\tau}(\alpha) \cdot i(\alpha, \nu) \cdot T w_{\tau}(\nu, \alpha)+O(i(\nu, \partial Q)), \\
& l_{\mathcal{L}}(\nu \cap X)=l_{\mathcal{L}}\left(\nu_{X}\right)+\frac{1}{2} \sum_{\alpha \in \partial Q} l_{\mathcal{L}}(\alpha) \cdot i(\alpha, \nu) \cdot T w_{\mathcal{L}}(\nu, \alpha)+O(i(\nu, \partial Q)) .
\end{aligned}
$$


By construction, $l_{\tau}\left(\nu_{X}\right)=l_{\mathcal{L}}\left(\nu_{X}\right)$.

$$
\begin{aligned}
& \left|l_{\tau}(\nu \cap X)-l_{\mathcal{L}}(\nu \cap X)\right| \\
\leq & \frac{1}{2} \sum_{\alpha \in \partial Q} l_{\tau}(\alpha) i(\nu, \alpha)\left|T w_{\tau}(\nu, \alpha)-T w_{\mathcal{L}}(\nu, \alpha)\right|+O(i(\nu, \partial Q)) .
\end{aligned}
$$

By construction, the Fenchel Nielsen twist coordinates for $\tau$ and $\mathcal{L}$ on any component $\alpha$ of $\partial Q$ coincide. Therefore, by Lemma 4.1, we have $\left|T w_{\tau}(\nu, \alpha)-T w_{\mathcal{L}}(\nu, \alpha)\right| \leq 4$. Thus

$$
\left|l_{\tau}(\nu \cap X)-l_{\mathcal{L}}(\nu \cap X)\right| \leq 2 \sum_{\alpha \in \partial Q} l_{\tau}(\alpha) \cdot i(\nu, \alpha)+O(i(\nu, \partial Q)) .
$$

Substituting this into Equation(25) and noting that we are working under the assumption that all components $\alpha$ of $\partial Q$ are extremely short in $\tau$, we obtain:

$$
l_{\mathcal{L}}\left(\nu_{t}^{+} \cap Q\right)+l_{\mathcal{L}}\left(\nu_{t}^{-} \cap Q\right)<l_{\tau}\left(\nu_{t}^{+} \cap Q\right)+l_{\tau}\left(\nu_{t}^{-} \cap Q\right)+O\left(l_{q}(\partial Q)\right) .
$$

Since every component of $\partial Q$ is extremely short in $\tau$, the collar lemma implies that $i(\nu, \partial Q) \stackrel{*}{\prec} l_{\tau}(\nu \cap Q)$ so we may replace the last approximation by

$$
l_{\mathcal{L}}\left(\nu_{t}^{+} \cap Q\right)+l_{\mathcal{L}}\left(\nu_{t}^{-} \cap Q\right) \stackrel{*}{\prec} l_{\tau}\left(\nu_{t}^{+} \cap Q\right)+l_{\tau}\left(\nu_{t}^{-} \cap Q\right) .
$$

Since for $\sigma=\mathcal{L}, \tau$ and $\nu=\nu_{t}^{ \pm}$, we have by Lemma 7.5

$l_{\sigma}(\nu \cap Q)=l_{\sigma}\left(\nu \cap Q_{T}\right)+l_{\sigma}\left(\nu \cap\left(Q \backslash Q_{T}\right)\right) \stackrel{*}{=} l_{\sigma}\left(\nu_{Q} \cap Q_{T}\right)+l_{\sigma}\left(\nu \cap\left(Q \backslash Q_{T}\right)\right)$,

we can apply Lemma 7.3 to subtract the contribution of the collars forming $Q \backslash Q_{T}$ from both sides to obtain:

$$
l_{\mathcal{L}}\left(\nu_{Q}^{+} \cap Q_{T}\right)+l_{\mathcal{L}}\left(\nu_{Q}^{-} \cap Q_{T}\right) \stackrel{*}{\prec} l_{\tau}\left(\nu_{Q}^{+} \cap Q_{T}\right)+l_{\tau}\left(\nu_{Q}^{-} \cap Q_{T}\right) .
$$

To complete the proof, we apply Lemmas 7.5 and 7.6:

$$
\begin{aligned}
& l_{\tau}\left(\nu_{Q}^{+} \cap Q_{T}\right)+l_{\tau}\left(\nu_{Q}^{-} \cap Q_{T}\right) \stackrel{*}{`} l_{g}\left(\nu_{Q}^{+} \cap Q_{T}\right)+l_{g}\left(\nu_{Q}^{-} \cap Q_{T}\right) \\
& \stackrel{*}{\asymp} l_{g}\left(\nu_{t}^{+} \cap Q_{T}\right)+l_{g}\left(\nu_{t}^{-} \cap Q_{T}\right) \text {. }
\end{aligned}
$$

7.3. Correspondence between thick components. In this section we show that the sets of short curves on $\mathcal{L}_{t}$ and $g_{t}$ coincide (Corollary 7.9) and prove Theorem 7.10 and Corollary 7.11 which, generalizing Theorem 3.9, shows that the geometries of the thick parts of $\mathcal{L}_{t}$ and $g_{t}$ are close. As in that proof, our strategy is to use short markings to estimate lengths. We begin with Proposition 7.7, which generalizes Proposition 3.6 to thick components. 
Proposition 7.7. Assume that $l_{\sigma}(\alpha)<\epsilon_{0}$ for every component $\alpha$ of $\partial Q$. Let $\rho>0$ and suppose that $l_{\sigma}(\zeta) \geq \rho$ for every non-peripheral simple closed curve $\zeta$ in $Q$. Then for any simple closed geodesic $\gamma$ on $(S, \sigma)$

$$
l_{\sigma}\left(\gamma \cap Q_{T}\right) \stackrel{*}{\star} i\left(\left.M_{\sigma}\right|_{Q}, \gamma\right),
$$

where the multiplicative constants depend only on $\rho$.

Proof. By Lemma 7.5 it is sufficient to prove that

$$
l_{\sigma}\left(\gamma_{Q} \cap Q_{T}\right) \stackrel{*}{*} i\left(\left.M_{\sigma}\right|_{Q}, \gamma\right) .
$$

We modify the argument in [18] Lemma 4.7.

Notice that since $Q_{T}$ is $\rho$-thick, it follows from Corollary 3.4 that the lengths of all the curves in $\left.M_{\sigma}\right|_{Q}$ are bounded above. Cutting $Q_{T}$ along the curves in $\left.M_{\sigma}\right|_{Q}$, we obtain a collection of convex polygons $\left\{D_{i}\right\}$, together with annuli $\left\{A_{j}\right\}$, where one boundary component $\partial_{0} A_{j}$ is a component of $\partial Q_{T}$, while the other component $\partial_{1} A_{j}$ is made up of arcs in $\left.M_{\sigma}\right|_{Q}$.

Curves in $\left.M_{\sigma}\right|_{Q}$ intersect at most twice, so by area considerations each $D_{i}$ has bounded diameter. We claim that the annuli $A_{j}$ also have bounded diameter. Since the length of $\partial_{0} A_{j}$ is bounded below by $\epsilon_{0}$, an area argument again shows that the distance between $\partial_{1} A_{j}$ and $\partial_{0} A_{j}$ is bounded above. Since, furthermore, the lengths of $\partial_{0} A_{j}$ and $\partial_{1} A_{j}$ are bounded above, it follows that $A_{j}$ has bounded diameter, as claimed. Setting $D=\max \left\{\operatorname{diam} D_{i}, \operatorname{diam} A_{j}\right\}$ gives the upper bound

$$
l_{\sigma}\left(\gamma_{Q} \cap Q_{T}\right) \leq i\left(\gamma,\left.M_{\sigma}\right|_{Q}\right) \cdot D .
$$

Since the lengths of all the curves in $\left.M_{\sigma}\right|_{Q}$ are bounded above, by the collar lemma, there is an embedded collar of definite radius $d$ around every curve in $\left.M_{\sigma}\right|_{Q}$. Therefore, if $\gamma$ crosses $\left.\beta \in M_{\sigma}\right|_{Q}$, then $l_{\sigma}(\gamma)>$ $d \cdot i(\gamma, \beta)$. Let $k$ be the number of pants curves in $Q$. Since there must be some $\left.\beta \in M_{\sigma}\right|_{Q}$ such that $i(\gamma, \beta) \geq i\left(\gamma,\left.M_{\sigma}\right|_{Q}\right) /(2 k)$, we have

$$
l_{\sigma}\left(\gamma_{Q} \cap Q_{T}\right)>\frac{d}{2 k} \cdot i\left(\gamma,\left.M_{\sigma}\right|_{Q}\right),
$$

giving the desired lower bound.

Applying Proposition 7.7, we can now deduce from Proposition 7.4 that a non-peripheral curve in $Q$ cannot be too short in $\mathcal{L}_{t}$. This is a preliminary step to showing the geometries of $\left(Q_{T}, \mathcal{L}_{t}\right)$ and $\left(Q_{T}, g_{t}\right)$ are close to each other.

Proposition 7.8. Let $Q$ be a component of $S \backslash \mathcal{S}_{\epsilon}\left(g_{t}\right)$ where $\epsilon$ is chosen as in Equation(24). Then for any non-peripheral simple closed curve $\zeta$ in $Q$, we have $l_{\mathcal{L}_{t}}(\zeta) \stackrel{*}{\succ} 1$. 
Proof. First, we claim that

$$
l_{g_{t}}\left(\nu_{t}^{+} \cap Q_{T}\right)+l_{g_{t}}\left(\nu_{t}^{-} \cap Q_{T}\right) \stackrel{*}{`} \lambda_{Q} .
$$

To see this, let $M_{g}$ be a short marking for $g_{t}$ and let $\left.M_{g}\right|_{Q}$ denote its restriction to $Q$. By Proposition 7.7, we have

$$
l_{g}\left(\nu_{t}^{+} \cap Q_{T}\right)+l_{g}\left(\nu_{t}^{-} \cap Q_{T}\right) \stackrel{*}{\smile} i\left(\left.M_{g}\right|_{Q}, \nu_{t}^{+}\right)+i\left(\left.M_{g}\right|_{Q}, \nu_{t}^{-}\right) \stackrel{*}{`} l_{q_{t}}\left(\left.M_{g}\right|_{Q}\right) .
$$

On the other hand, $l_{g_{t}}\left(\left.M_{g}\right|_{Q}\right) \stackrel{*}{=}$. Hence by Theorem 5.5

$$
l_{q_{t}}\left(\left.M_{g}\right|_{Q}\right) \stackrel{*}{`} \lambda_{Q}
$$

and the claim is proved.

Now if $\zeta$ is a non-peripheral simple closed curve in $Q$ with $l_{\mathcal{L}_{t}}(\zeta)<\epsilon_{0}$, then consideration of the collar about $\zeta$ gives the estimate

$$
l_{\mathcal{L}_{t}}\left(\nu \cap Q_{T}\right) \stackrel{*}{\succ} i(\nu, \zeta) \log \frac{1}{l_{\mathcal{L}_{t}}(\zeta)}
$$

for any $\nu \in \mathcal{M L}(S)$, so in particular,

$$
l_{\mathcal{L}_{t}}\left(\nu_{t}^{+} \cap Q_{T}\right)+l_{\mathcal{L}_{t}}\left(\nu_{t}^{-} \cap Q_{T}\right) \stackrel{*}{\succ} l_{q_{t}}(\zeta) \log \frac{1}{l_{\mathcal{L}_{t}}(\zeta)} .
$$

Proposition 7.4 and Equation $(27)$ give $\lambda_{Q} \stackrel{*}{\succ} l_{q}(\zeta) \log \left[1 / l_{\mathcal{L}_{t}}(\zeta)\right]$. From the definition of $\lambda_{Q}$ we have $\lambda_{Q} / l_{q}(\zeta) \leq 1$, so that $l_{\mathcal{L}_{t}}(\zeta) \stackrel{*}{\succ} 1$.

Proposition 7.8 shows that the sets of extremely short curves on $\mathcal{L}_{t}$ and $g_{t}$ coincide. More precisely:

Corollary 7.9. Let $\epsilon$ be as in Equation(24). Then there exists $\epsilon^{\prime}>0$ such that $\mathcal{S}_{\epsilon^{\prime}}\left(\mathcal{L}_{t}\right) \subset \mathcal{S}_{\epsilon}\left(g_{t}\right)$.

Finally, we prove the main results of this section.

Theorem 7.10. Let $Q$ be a component of $S \backslash \mathcal{S}_{\epsilon}\left(g_{t}\right)$ which is not a pair of pants, and let $M_{\mathcal{L}_{t}}$ be a short marking for $\mathcal{L}_{t}$. Then

$$
l_{g_{t}}\left(\left.M_{\mathcal{L}_{t}}\right|_{Q}\right) \stackrel{*}{=} 1 .
$$

Proof. By Theorem 5.5, we have

$$
l_{g_{t}}\left(\left.M_{\mathcal{L}_{t}}\right|_{Q}\right) \stackrel{*}{`} \frac{1}{\lambda_{Q}} l_{q_{t}}\left(\left.M_{\mathcal{L}_{t}}\right|_{Q}\right) \stackrel{*}{`} \frac{1}{\lambda_{Q}}\left[i\left(\left.M_{\mathcal{L}_{t}}\right|_{Q}, \nu^{+}\right)+i\left(\left.M_{\mathcal{L}_{t}}\right|_{Q}, \nu^{-}\right)\right] .
$$

By Proposition 7.8, there is a constant $\rho=\rho\left(\epsilon_{0}\right)$ depending only on $\epsilon_{0}$ such that $l_{\mathcal{L}_{t}}(\zeta)>\rho\left(\epsilon_{0}\right)$ for every non-peripheral curve $\zeta$ in $Q$. Therefore, we can apply Proposition 7.7 to get

$$
i\left(\left.M_{\mathcal{L}_{t}}\right|_{Q}, \nu_{t}^{+}\right)+i\left(\left.M_{\mathcal{L}_{t}}\right|_{Q}, \nu_{t}^{-}\right) \stackrel{*}{\asymp} l_{\mathcal{L}_{t}}\left(\nu_{t}^{+} \cap Q_{T}\right)+l_{\mathcal{L}_{t}}\left(\nu_{t}^{-} \cap Q_{T}\right) .
$$

Since the lower bound $l_{g_{t}}\left(\left.M_{\mathcal{L}_{t}}\right|_{Q}\right) \stackrel{*}{\succ} 1$ is trivial, the result now follows from Proposition 7.4 and Equation(27). 
Equivalently, we can formulate Theorem 7.10 in terms of the surface $Q_{0}$ obtained from $Q$ by replacing every boundary component with a puncture. Let $\left.\mathcal{L}_{t}\right|_{Q_{0}}$ and $\left.g_{t}\right|_{Q_{0}}$ be respectively, the surface $Q_{0}$ equipped with the metrics obtained from $\mathcal{L}_{t}$ and $g_{t}$ by pinching the curves in $\partial Q$ but otherwise leaving the metric unchanged. Then we have:

Corollary 7.11. Let $Q$ be a component of $S \backslash \mathcal{S}_{\epsilon}\left(g_{t}\right)$. Then

$$
d_{\mathcal{T}\left(Q_{0}\right)}\left(\left.\mathcal{L}_{t}\right|_{Q_{0}},\left.g_{t}\right|_{Q_{0}}\right)=O(1) .
$$

Proof. The boundary components of both $\left(Q, \mathcal{L}_{t}\right)$ and $\left(Q, g_{t}\right)$ are extremely short. In this case, it was shown in [19] (see the proof of Lemma 7.6) that $\left(Q, \mathcal{L}_{t}\right)$ and $\left(Q, g_{t}\right)$ can be embedded into $\left(Q_{0},\left.\mathcal{L}_{t}\right|_{Q_{0}}\right)$ and $\left(Q_{0},\left.g_{t}\right|_{Q_{0}}\right)$ respectively, by a $K$-quasi-conformal map, where $K$ depends only on $\epsilon_{0}$. Since simple curves do not penetrate the thin part of $Q_{0}$, the above theorem combined with the last paragraph of the proof of Theorem 3.9, gives the result.

7.4. Comparison of lengths of short curves. As a consequence of Theorem 7.10, we can show:

Proposition 7.12. Let $\alpha$ be an extremely short curve on $\mathcal{L}_{t}$. Then

$$
H_{t}(\alpha) \prec K_{t}(\alpha) \text {. }
$$

Proof. With $\mathcal{B}$ as in Equation(12), let $\beta \in \mathcal{B}$ be the curve that has the largest $q_{t}$-length, so that

$$
H_{t}(\alpha) \stackrel{*}{\asymp} \frac{l_{q_{t}}(\beta)}{l_{q_{t}}(\alpha)} .
$$

Since $\mathcal{S}_{\epsilon}\left(g_{t}\right) \subset \mathcal{S}_{\epsilon_{0}}\left(\mathcal{L}_{t}\right)$, it follows that the curves in $\mathcal{P}_{\mathcal{L}_{t}}$ are disjoint from $S_{\epsilon}\left(g_{t}\right)$. Thus, $\alpha$ and $\beta$ are contained in a common component $Q$ of $S \backslash S_{\epsilon}\left(g_{t}\right)$.

Suppose first that $\beta$ is not peripheral in $Q$. Then $\left.\beta \in M_{\mathcal{L}}\right|_{Q}$, so that by Theorem 5.5 and 7.10 ,

$$
l_{q}(\beta) \stackrel{*}{`} \lambda_{Q} l_{g}(\beta) \stackrel{*}{ } \lambda_{Q} .
$$

If in addition, $\alpha$ is not peripheral, then $l_{q}(\alpha) \stackrel{*}{`} \lambda_{Q}$ so that $H(\alpha) \stackrel{*}{=} 1$ and the desired inequality holds trivially. If $\alpha$ is peripheral, then

$$
H(\alpha) \stackrel{*}{`} \frac{l_{q}(\beta)}{l_{q}(\alpha)} \stackrel{*}{\asymp} \frac{\lambda_{Q}}{l_{q}(\alpha)} \leq K(\alpha) .
$$

Now suppose that $\beta$ is peripheral in $Q$. If $Q$ is a pair of pants, then the desired inequality follows from the definition of $H(\alpha)$ and $K(\alpha)$. If $Q$ is not a pair of pants, then since the component of $\left.Q \backslash M_{g}\right|_{Q}$ containing $\beta$ is an annulus whose one boundary component is $\beta$ and 
the other a finite (at most 4) union of arcs coming from curves $\cup \gamma_{i}$ in $\left.M_{g}\right|_{Q}$, again by Theorem 5.5 we obtain

$$
l_{q}(\beta) \leq \sum l_{q}\left(\gamma_{i}\right) \stackrel{*}{`} \sum \lambda_{Q} l_{g}\left(\gamma_{i}\right) \stackrel{*}{ } \lambda_{Q},
$$

from which the result follows as before.

We summarize the length estimate that follows from Theorem 6.1, Proposition 7.1, and Proposition 7.12:

Theorem 7.13. Let $\alpha$ be any curve on $S$ which is neither vertical nor horizontal. If $\alpha$ is extremely short in $\mathcal{L}_{t}$, then

$$
\frac{1}{l_{\mathcal{L}_{t}}(\alpha)} \asymp \max \left\{D_{t}(\alpha), \sqrt{K_{t}(\alpha)}\right\} .
$$

In case $\alpha$ is vertical or horizontal, we have

Theorem 7.14. If $\alpha$ is vertical, then

$$
\frac{1}{l_{\mathcal{L}_{t}}(\alpha)} \asymp \max \left\{e^{-2 t} \operatorname{Mod} F_{0}(\alpha), \sqrt{K_{t}(\alpha)}\right\} .
$$

If $\alpha$ is horizontal, then the estimate is the same except that the first term is replaced by $e^{2 t} \operatorname{Mod} F_{0}(\alpha)$.

Proof. There are multiplicative constants depending only on the fixed laminations $\nu^{ \pm}$such that

$$
\frac{m^{\mp}(\alpha)}{i\left(\nu^{ \pm}, \alpha\right)} \stackrel{*}{`} \operatorname{Mod} F_{0}(\alpha)
$$

(see Theorems 6.8 and 5.11) holds independently of $\alpha$ in a tautological way, due to the fact that the total number of vertical (or horizontal) curves is finite; it is bounded above by $-\chi(S)$. The proofs of Proposition 7.1 and 7.12 go through in this case, so that $H_{t}(\alpha) \asymp K_{t}(\alpha)$. Hence the estimate follows from Theorem 6.8.

7.5. Teichmüller distance. With the preceding collection of results at our disposal, computing the Teichmüller distance between $\mathcal{G}_{t}$ and $\mathcal{L}_{t}$ becomes an easy application of Minsky's product region theorem 4.5.

Theorem 7.15. The Teichmüller distance between $\mathcal{G}_{t}$ and $\mathcal{L}_{t}$ is given by

$$
d_{\mathcal{T}(S)}\left(\mathcal{G}_{t}, \mathcal{L}_{t}\right)=\max _{\alpha \in \mathcal{S}_{\epsilon}\left(g_{t}\right)} \log \frac{l_{g_{t}}(\alpha)}{l_{\mathcal{L}_{t}}(\alpha)} \pm O(1)
$$

Proof. As noted before, $l_{\mathcal{L}_{t}}(\alpha) \leq \epsilon_{0}$ for every $\alpha \in \mathcal{S}_{\epsilon}\left(g_{t}\right)$. To simplify notation, let $\mathcal{E}_{t}=\mathcal{S}_{\epsilon}\left(g_{t}\right)$. By Theorem 4.5, we have

$d_{\mathcal{T}(S)}\left(\mathcal{G}_{t}, \mathcal{L}_{t}\right)=\max _{\alpha \in \mathcal{E}_{t}}\left\{d_{\mathcal{T}\left(S_{\mathcal{E}_{t}}\right)}\left(\Pi_{0}\left(\mathcal{G}_{t}\right), \Pi_{0}\left(\mathcal{L}_{t}\right)\right), d_{\mathbb{H}_{\alpha}}\left(\Pi_{\alpha}\left(\mathcal{G}_{t}\right), \Pi_{\alpha}\left(\mathcal{L}_{t}\right)\right)\right\} \pm O(1)$, 
where $S_{\mathcal{E}_{t}}$ is the surface obtained from $S$ by removing $\mathcal{E}_{t}$ and replacing the resulting boundary components by punctures and $\Pi_{0}, \Pi_{\alpha}$ are defined as in Section 4.4. From Corollary 7.11 we deduce that

$$
d_{\mathcal{T}\left(S_{\mathcal{E}}\right)}\left(\Pi_{0}\left(\mathcal{G}_{t}\right), \Pi_{0}\left(\mathcal{L}_{t}\right)\right)=O(1)
$$

If $t-t_{\alpha}>0$, then by Theorem 5.10 or 5.12 we have $T w_{g_{t}}\left(\nu^{+}, \alpha\right) l_{g_{t}}(\alpha)=$ $O(1)$ and by Theorem 6.2 or 6.9 we have $T w_{\mathcal{L}_{t}}\left(\nu^{+}, \alpha\right) l_{\mathcal{L}_{t}}(\alpha)=O(1)$. Applying Corollary 4.7 to $g_{t}$ and $\mathcal{L}_{t}$ and the lamination $\nu^{+}$, we find

$$
\exp d_{\mathbb{H}_{\alpha}}\left(\Pi_{\alpha}\left(\mathcal{G}_{t}\right), \Pi_{\alpha}\left(\mathcal{L}_{t}\right)\right) \stackrel{*}{=} l_{g_{t}}(\alpha) / l_{\mathcal{L}_{t}}(\alpha) .
$$

If $t-t_{\alpha}<0$, the same result holds by applying a similar argument with $\nu^{-}$.

The combinatorial nature of our length estimates allows us to use Theorem 7.15 to construct examples in which $\mathcal{L}$ and $\mathcal{G}$ have a variety of different relative behaviors. As a special case, if $S$ is a once-punctured torus or four-times-punctured sphere, every thick component must be a pair of pants. In this case, $K_{t}(\alpha)$ is bounded and therefore, a curve gets short only if $d_{\alpha}\left(\nu^{+}, \nu^{-}\right)$is large:

Corollary 7.16. If $S$ is a once-punctured torus or a four-times punctured sphere, then for any measured laminations $\nu^{+}, \nu^{-}$, the associated Teichmüller geodesic and line of minima satisfies

$$
d_{\mathcal{T}(S)}\left(\mathcal{G}_{t}, \mathcal{L}_{t}\right)=O(1)
$$

However, on surfaces of higher genus, it is possible to have $\nu^{+}, \nu^{-}$ and $\alpha$ such that $d_{\alpha}\left(\nu^{+}, \nu^{-}\right)$is bounded while $K_{t}(\alpha)$ is arbitrarily large. We can construct a simple example as follows. Take two Euclidean squares each of area $1 / 2$ and cut open a slit of length $\varepsilon$ at each of their centers. Although it is not necessary, for concreteness we can assume that in both squares, the slit is parallel to a pair of sides. Foliate each
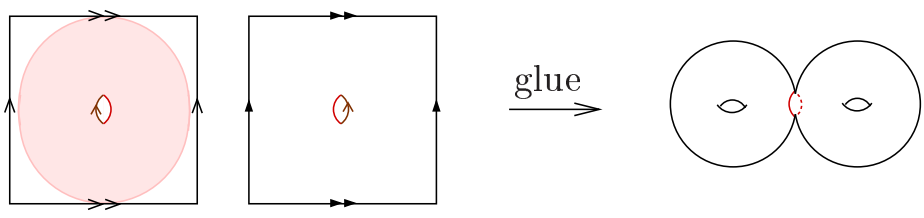

Figure 4. Glue two slit tori along slit.

square by the two mutually orthogonal foliations that both make angle $\pi / 4$ with the slit and for each, take the transverse measure induced by the Euclidean metric. Identify pairs of sides in each square to obtain two one-holed tori $T_{1}, T_{2}$ and glue $T_{1}, T_{2}$ tori along their boundaries, 
as shown in the figure, to obtain a genus two surface $S$ with waist curve $\alpha$. The two foliations match along $\alpha$ and specifying one to be the vertical foliation defines a quadratic differential $q=q_{0}$ on $S$. Let $\mathcal{G}$ and $\mathcal{L}$ be respectively, the Teichmüller geodesic and the line of minima defined by the vertical and horizontal foliations $\nu^{-}$and $\nu^{+}$of $q_{0}$. In this example, the foliations are rational, but it is easy to see that varying the initial angle of the slit gives more general foliations.

Let $q_{t}$ be the associated family of quadratic differentials. Note that at time $t=0$ the curve $\alpha$ is balanced. The $q_{t}$-geodesic representative of $\alpha$ is unique and the flat annulus corresponding to $\alpha$ is degenerate. Thus, by Proposition 5.6, we have $d_{\alpha}\left(\nu^{+}, \nu^{-}\right)=O(1)$. But since $\lambda_{T_{1}}=\lambda_{T_{2}}=$ $1 / \sqrt{2}$ at $t=0$, we have $K_{0}(\alpha) \asymp 1 / \varepsilon$ (the shaded region indicates a maximal expanding annulus). Assuming $\varepsilon$ is very small, Theorems 5.8 and 6.1 give

$$
\frac{1}{l_{g_{0}}(\alpha)} \asymp \log \frac{1}{\varepsilon} \text { and } \frac{1}{l_{\mathcal{L}_{0}}(\alpha)} \asymp \frac{1}{\sqrt{\varepsilon}} .
$$

Thus by Theorem 7.15,

$$
d_{\mathcal{T}(S)}\left(\mathcal{G}_{0}, \mathcal{L}_{0}\right) \succ \log \frac{l_{g_{0}}(\alpha)}{l_{\mathcal{L}_{0}}(\alpha)} \asymp \log \frac{1}{\sqrt{\varepsilon} \log [1 / \varepsilon]} .
$$

In fact, because for any two hyperbolic metrics $\sigma, \tau$ we have [27]

$$
d_{\mathcal{T}(S)}(\sigma, \tau) \geq \sup _{\zeta \in \mathcal{S}} \frac{l_{\sigma}(\zeta)}{l_{\tau}(\zeta)}
$$

and because the length of $\alpha$ along $\mathcal{G}$ is (coarsely) shortest at the balance time $t_{\alpha}=0$ [20], the following stronger inequality holds:

$$
\inf _{t \in \mathbb{R}} d_{\mathcal{T}(S)}\left(\mathcal{G}_{t}, \mathcal{L}_{0}\right) \geq \inf _{t \in \mathbb{R}} \log \frac{l_{g_{t}}(\alpha)}{l_{\mathcal{L}_{0}}(\alpha)} \succ \log \frac{l_{g_{0}}(\alpha)}{l_{\mathcal{L}_{0}}(\alpha)} .
$$

Taking $\varepsilon$ small enough we can ensure that $\mathcal{L}_{0}$ is as far as we like from any point on $\mathcal{G}$. This example can be easily extended to any surface of large complexity, by which we mean a surface whose genus $g$ and number of punctures $p$ satisfies $3 g-4+p \geq 1$. In summary,

Corollary 7.17. If $S$ is a surface of large complexity, then given any $n>0$, there are measured laminations $\nu^{+}(n), \nu^{-}(n)$ on $S$ which depend on $n$, such that for the associated Teichmüller geodesic $\mathcal{G}(n)$ and line of minima $\mathcal{L}(n)$,

$$
\inf _{t \in \mathbb{R}} d_{\mathcal{T}(S)}\left(\mathcal{G}_{t}(n), \mathcal{L}_{0}(n)\right)>n .
$$

It is also possible to construct examples for any such surface where the two measured laminations are fixed and the associated Teichmüller geodesic and line of minima satisfy $d_{\mathcal{T}(S)}\left(\mathcal{G}_{t_{n}}, \mathcal{L}_{t_{n}}\right)>n$ for a sequence 
of times $t_{n} \rightarrow \infty$ as $n \rightarrow \infty$. This however, is beyond the scope of this paper.

\section{ApPEndiX}

We give proofs of the length estimates that were deferred in previous sections.

Proof of Lemma 3.3. Let $H$ be one of the two right-angled hexagons obtained by cutting $P$ along the three seams, i.e., common perpendiculars between $\alpha_{1}, \alpha_{2}, \alpha_{3}$. Let $l_{i}=l\left(\alpha_{i}\right) / 2$ and $d_{i}$ be the length of the common perpendiculars, labeled as shown in Figure 5. By the cosine

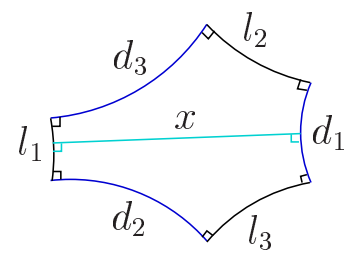

Figure 5. Half a pair of pants.

formula for right-angled hexagons, we have

$$
\cosh d_{3}=\frac{\cosh l_{3}+\cosh l_{1} \cosh l_{2}}{\sinh l_{1} \sinh l_{2}}
$$

By hypothesis, $l_{i}<L / 2$, so $\sinh l_{i} \stackrel{*}{=} l_{i}$ and $\cosh l_{i} \stackrel{*}{=} 1$, where the multiplicative constants involved depend only on $L$. Therefore,

$$
\cosh d_{3} \stackrel{*}{\longleftarrow} \frac{1}{l_{1} l_{2}} .
$$

It follows that $d_{3}=\log \left[1 / l_{1}\right]+\log \left[1 / l_{2}\right] \pm O(1)$, where the bound on the additive error depends only on $L$.

Now we estimate the length of the perpendicular from $\alpha_{i}$ to itself. Let $x$ be the length of the perpendicular as in Figure 5. By the formula for right-angled pentagons, we have

$$
\cosh x=\sinh l_{2} \sinh d_{3} .
$$

Since $\sinh l_{2} \stackrel{*}{\asymp} l_{2}$ and by the above, $\sinh d_{3} \stackrel{*}{=} 1 /\left[l_{1} l_{2}\right]$, it follows that $\cosh x \stackrel{*}{=} 1 / l_{1}$. Hence, $x=\log \left[1 / l_{1}\right] \pm O(1)$. Since $P$ is made of two isometric copies of $H$, we obtain the desired estimate.

To prove Lemma 6.3, in addition to the standard hexagon and pentagon formulae (see for example [1] or [23]), we need the following 
expression for derivatives of side lengths derived in [23] Proposition 2.3:

Lemma 8.1. Let $H$ be a planar right-angled hexagon with sides labeled $i=1, \ldots, 6$ in cyclic order about $\partial H$. Let $l_{i}$ denote the length of side $i$ and for $n \operatorname{Mod} 6$, let $p_{n, n+3}$ be the perpendicular distance from side $n$ to side $n+3$. Letting' denote derivative with respect to some variable $x$ :

$$
\left(\cosh p_{n, n+3}\right) l_{n}^{\prime}=l_{n+3}^{\prime}-\left(\cosh l_{n-2}\right) l_{n-1}^{\prime}-\left(\cosh l_{n+2}\right) l_{n+1}^{\prime} \text {. }
$$

It is convenient to subdivide Lemma 6.3 into two parts, Lemma 8.2 and Lemma 8.3, depending on whether or not the common perpendicular in question is adjacent to $\alpha$. We begin with a somewhat more detailed discussion of the possible configurations.

Let $P$ be a pair of pants in $S \backslash \mathcal{P}_{\mathcal{L}_{t}}$ that has $\alpha$ as a boundary component. For clarity, we distinguish between the three boundary curves $\alpha, \beta, \gamma$ of $P$ and their projections $\pi(\alpha), \pi(\beta), \pi(\gamma)$ to $S$. We may always assume that $\pi(\gamma) \neq \pi(\alpha)$. There are then two possible configurations depending on whether or not $\pi(\beta)=\pi(\alpha)$. Figure 6(a) represents the case in which $\pi(\beta)=\pi(\alpha)$ and Figure $6(\mathrm{~b})$, the case in which $\pi(\beta) \neq \pi(\alpha)$. In (b), we do not rule out the possibility that $\pi(\beta)=\pi(\gamma)$. This leads to a dichotomy in the formulae used in the proofs, but not in the final estimates. Let $d$ be the length of the perpendicular between $\alpha$ and $\gamma$ and let $h_{\alpha}$ be the length of the shortest perpendicular from $\alpha$ to itself.

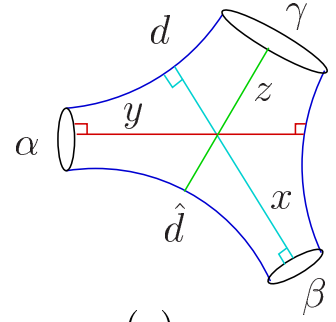

(a)

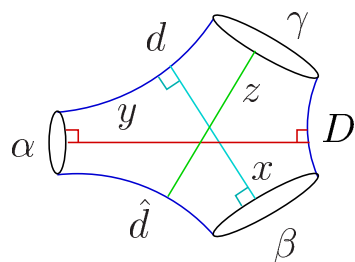

(b)

Figure 6. (a) $\pi(\alpha)=\pi(\beta)$; (b) $\pi(\alpha) \neq \pi(\beta)$.

Lemma 8.2. Suppose that $\alpha$ is extremely short in $\mathcal{L}_{t}$. Then the derivatives of the perpendiculars adjacent to $\alpha$ are as follows:

$$
\begin{aligned}
\text { (i) } d^{\prime}=\frac{\partial d}{\partial l(\alpha)} \stackrel{*}{`}-\frac{1}{l(\alpha)} . \\
\text { (ii) } h_{\alpha}^{\prime}=\frac{\partial h_{\alpha}}{\partial l(\alpha)} \stackrel{*}{\rightleftharpoons}-\frac{1}{l(\alpha)} .
\end{aligned}
$$


Proof. Let $x, y, z$ be the lengths of the perpendiculars as shown in Figure 6.

(i) In case (a), the formula (28) and the pentagon formula ([23] lemma 2.1) give, respectively,

$$
d^{\prime} \cosh x=-\frac{\cosh \hat{d}}{2}, \quad \cosh x=\sinh \hat{d} \sinh \frac{l(\alpha)}{2} .
$$

If $l(\alpha)$ is small, then $\sinh l(\alpha) \stackrel{*}{\asymp} l(\alpha)$ and by Lemma 3.3, $\hat{d} \succ 1 / \log l(\alpha)$ so that $\operatorname{coth} \hat{d}=O(1)$. Hence

$$
d^{\prime}=-\frac{\operatorname{coth} \hat{d}}{\sinh [l(\alpha) / 2]} \stackrel{*}{=}-\frac{1}{l(\alpha)} .
$$

In case (b), using the same formulae as above, we get

$$
d^{\prime} \cosh x=\frac{1-\cosh \hat{d}}{2}, \quad \cosh x=\sinh \hat{d} \sinh \frac{l(\alpha)}{2} .
$$

Now by Lemma 3.3, $\hat{d}=2 \log [1 / l(\alpha)] \pm O(1)$ and therefore $\sinh \hat{d} \stackrel{*}{ }$ $1 / l(\alpha)^{2} \stackrel{*}{\asymp} \cosh \hat{d}$. Hence,

$$
d^{\prime}=\frac{1-\cosh \hat{d}}{2 \sinh \hat{d} \sinh [l(\alpha) / 2]} \stackrel{*}{`} l(\alpha)[1-\cosh \hat{d}] \stackrel{*}{ }-\frac{1}{l(\alpha)} .
$$

(ii) In case (a), $h_{\alpha}=2 y$ and $\cosh y=\sinh d \sinh [l(\gamma) / 2]$. Differentiating both sides, we get

$$
y^{\prime}=\frac{\sinh [l(\gamma) / 2] \cosh d}{\sinh y} d^{\prime}
$$

By Lemma 3.3, $\cosh d \stackrel{*}{ } 1 /[l(\alpha) l(\gamma)]$ and $\sinh y \stackrel{*}{ } 1 / l(\alpha)$, so we obtain $y^{\prime} \stackrel{*}{ }-1 / l(\alpha)$, as desired.

In case (b), $h_{\alpha}=\hat{d}$ and by [23] equation(6),

$$
\hat{d}^{\prime} \cosh z=-\cosh d \text {. }
$$

Substituting $\cosh z \stackrel{*}{ } 1 / l(\gamma)$ and $\cosh d \stackrel{*}{ } 1 /[l(\alpha) l(\gamma)]$ gives the desired estimate.

Now consider perpendiculars in $P$ that are disjoint from $\alpha$. Let $h_{\gamma}$ be the length of the perpendicular from $\gamma$ to itself, as shown in Figure 7. Further, if $\pi(\beta) \neq \pi(\alpha)$, let $D$ be the length of the perpendicular between $\beta, \gamma$. We have $h_{\gamma}=D$ when $\pi(\beta)=\pi(\gamma)$ (see Figure $7(\mathrm{~b})$ ). 


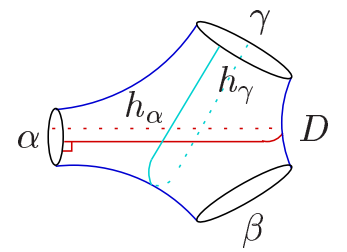

(a)

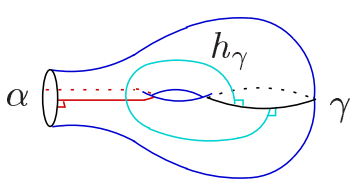

(b)

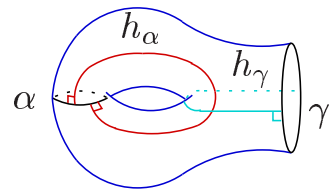

(c)

Figure 7. (a) $\pi(\alpha), \pi(\beta), \pi(\gamma)$ all distinct; (b) $\pi(\beta)=$ $\pi(\gamma) ;(\mathrm{c}) \pi(\alpha)=\pi(\beta)$

Lemma 8.3. Suppose that $\alpha$ is extremely short in $\mathcal{L}_{t}$. Then the derivatives of the perpendiculars not adjacent to $\alpha$ are as follows:

$$
\begin{aligned}
& \text { (i) } h_{\gamma}^{\prime}=\frac{\partial h_{\gamma}}{\partial l(\alpha)} \stackrel{*}{ } \quad l(\alpha) . \\
& \text { (ii) } D^{\prime}=\frac{\partial D}{\partial l(\alpha)} \stackrel{*}{ } \quad l(\alpha) .
\end{aligned}
$$

Proof. (i) Assume that $\pi(\beta) \neq \pi(\gamma)$ so that we are in the configuration of Figure 7 (a) or (c). Consider the 'front' hexagon in $P$ and denote the lengths of the sides as shown in Figure 8, so that $l_{1}=l(\alpha) / 2, l_{2}=l(\gamma) / 2$, and $z=h_{\gamma} / 2$. By the pentagon formula,

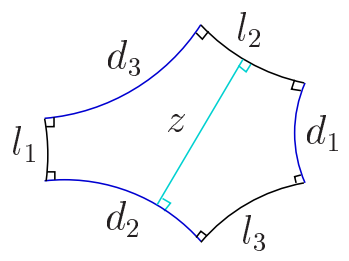

FiguRE 8. Front right-angled hexagon.

$\cosh z=\sinh d_{3} \sinh l_{1}$. Taking the derivative with respect to $l_{1}$, we get

$$
z^{\prime} \sinh z=d_{3}^{\prime} \cosh d_{3} \sinh l_{1}+\sinh d_{3} \cosh l_{1} .
$$

In the proof of Lemma 8.2, we had $d_{3}^{\prime}=-\operatorname{coth} d_{2} / \sinh l_{1}$, and by the cosine formula for right-angled hexagons, we have

$$
\cosh l_{1}=\frac{\cosh d_{1}+\cosh d_{2} \cosh d_{3}}{\sinh d_{2} \sinh d_{3}} .
$$

Substituting these, we get

$$
z^{\prime}=\frac{1}{\sinh z} \cdot \frac{\cosh d_{1}}{\sinh d_{2}}
$$


Now by the sine formula for right-angled hexagons,

$$
\frac{1}{\sinh d_{2}}=\frac{\sinh l_{1}}{\sinh d_{1} \sinh l_{2}} \text {. }
$$

With this, we have

$$
z^{\prime}=\sinh l_{1} \cdot \frac{\cosh d_{1}}{\sinh d_{1}} \cdot \frac{1}{\sinh l_{2} \sinh z} \stackrel{*}{`} \sinh l_{1} \stackrel{*}{=} l_{1},
$$

since $\operatorname{coth} d_{1} \stackrel{*}{=} 1$ and $\sinh z \stackrel{*}{=} 1 / l_{2}$ when $l_{1}, l_{2}$ are respectively, bounded.

In the case $\pi(\beta)=\pi(\gamma)$, we have $h_{\gamma}^{\prime}=D^{\prime}$, which is computed below. (ii) Let $y$ be the length of the perpendicular between $\alpha$ and the common perpendicular of $\beta, \gamma$, as in Figure 6. Then by Equation (28) and the pentagon formula, we have

$$
D^{\prime} \cosh y=1, \quad \cosh y=\sinh d \sinh \frac{l(\gamma)}{2} .
$$

By Lemma 3.3, $d=\log [1 / l(\alpha)]+\log [1 / l(\gamma)] \pm O(1)$ and therefore $\sinh d \stackrel{*}{\asymp} 1 /[l(\alpha) l(\gamma)]$. Since the pants system is $\operatorname{short,} \sinh [l(\gamma) / 2] \stackrel{*}{ }$ $l(\gamma)$. Thus $D^{\prime} \stackrel{*}{\asymp} l(\alpha)$, as claimed.

Lemmas 8.2 and 8.3 together prove Lemma 6.3.

Proof of Lemma 7.2. If $\xi$ has a component $\zeta$ contained in $\partial Q$, then $\zeta \cap Q=\zeta=\zeta_{Q}$, which has no effect on the inequality. Thus, we assume that no component of $\xi$ is contained in $\partial Q$. For simplicity, let us further assume that $\xi$ is a simple closed curve. Since both sides of the inequality scale linearly with weights, it is sufficient to prove the lemma under this assumption. The basic idea is to approximate an arc $\eta$ of $\xi \cap Q$ with the union of $\eta_{Q}$ and segments $p \hat{p}, q \hat{q}$ which run along $\partial P$ between the endpoints $\hat{p}, \hat{q}$ of $\eta_{Q}$ and the corresponding endpoints $p, q$ of $\eta$. Let $\alpha_{p}, \alpha_{q}$ denote the components of $\partial P$ that contain $p, q$, respectively. It is possible that $\alpha_{p}=\alpha_{q}$.

We will show that there are uniform constants $C, C^{\prime}$ such that

$$
\begin{aligned}
& \left|l_{\sigma}(p \hat{p})-l_{\sigma}\left(\alpha_{p}\right) \cdot T w_{\sigma}\left(\xi, \alpha_{p}\right) / 2\right|<C \\
& \left|l_{\sigma}(q \hat{q})-l_{\sigma}\left(\alpha_{q}\right) \cdot T w_{\sigma}\left(\xi, \alpha_{q}\right) / 2\right|<C \\
& \left|l_{\sigma}(\eta)-\left[l_{\sigma}(p \hat{p})+l_{\sigma}\left(\eta_{Q}\right)+l_{\sigma}(q \hat{q})\right]\right|<C^{\prime} .
\end{aligned}
$$

It is convenient to consider the picture in the universal cover $\mathbb{H}^{2}$, as shown in Figure 9. Let $\tilde{\eta}$ be a lift of $\eta$ and let $\tilde{\alpha}_{p}, \tilde{\alpha}_{q}$ be the lifts of $\alpha_{p}, \alpha_{q}$ that contain the endpoints of $\tilde{\eta}$. Since $\eta_{Q}$ is homotopic to $\eta$ relative to $\partial Q$ and is perpendicular to $\partial Q$, its lift $\tilde{\eta}_{Q}$ is the unique perpendicular between $\tilde{\alpha_{p}}, \tilde{\alpha_{q}}$, drawn as the segment $\hat{p} \hat{q}$ in the figure. There are two possible cases, depending on whether or not $\tilde{\eta}$ intersects $\tilde{\eta}_{Q}$. 

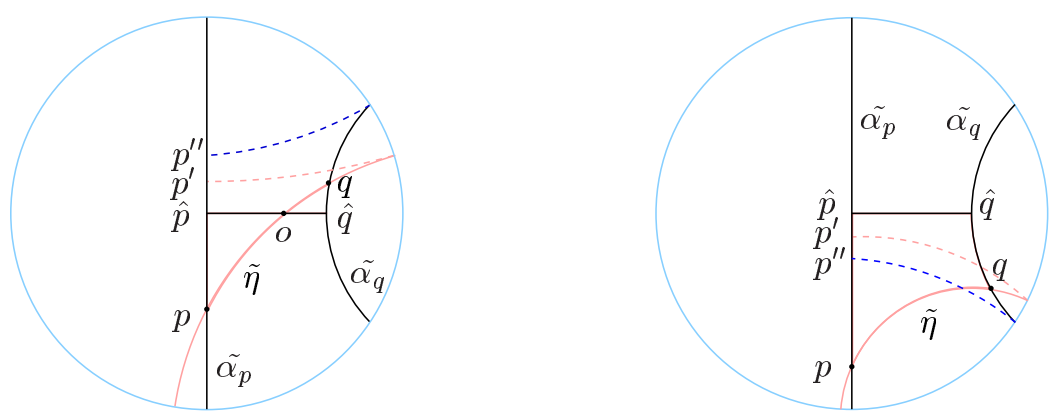

Figure 9. Approximating the length of $\tilde{\eta}$.

Let $p^{\prime}, p^{\prime \prime}$ be the feet of the perpendiculars as shown. That is, $p^{\prime}$ is the foot of the projection from the geodesic ray extending $\tilde{\eta}$ to $\tilde{\alpha}_{p}$ and $p^{\prime \prime}$ is the foot of the projection from $\tilde{\alpha}_{q}$ to $\tilde{\alpha}_{p}$. In either case, by definition of twist,

$$
T w_{\sigma}\left(\xi, \alpha_{p}, p\right)=2 l_{\sigma}\left(p p^{\prime}\right) / l_{\sigma}\left(\alpha_{p}\right)
$$

and furthermore,

$$
\left|l_{\sigma}(p \hat{p})-l_{\sigma}\left(p p^{\prime}\right)\right|<l_{\sigma}\left(\hat{p} p^{\prime \prime}\right) .
$$

On the other hand, by trigonometry in $\mathbb{H}^{2}$, we have

$$
\cosh l_{\sigma}\left(\hat{p} p^{\prime \prime}\right)=1 / \tanh l_{\sigma}(\hat{p} \hat{q})=1 / \tanh l_{\sigma}\left(\eta_{Q}\right) .
$$

Since $\eta_{Q}$ goes from $\alpha_{p}$ to $\alpha_{q}$ and since by hypothesis $l_{\sigma}\left(\alpha_{p}\right), l_{\sigma}\left(\alpha_{q}\right)<\ell$, it follows from the collar lemma that $l_{\sigma}\left(\eta_{Q}\right)>c(\ell)$ for some constant $c(\ell)$ depending only on $\ell$. This implies that there is a constant $C=C(\ell)$ depending only on $\ell$ such that $l_{\sigma}\left(\hat{p} p^{\prime \prime}\right)<C(\ell)$. Therefore, Equation (31) gives Equation (29), as desired. Of course, the same argument applies to $\alpha_{q}$ so

$$
\left|l_{\sigma}(q \hat{q})-l_{\sigma}\left(\alpha_{q}\right) \cdot T w_{\sigma}\left(\xi, \alpha_{q}, q\right) / 2\right|<C .
$$

To show Equation (30), we use the well known fact that for any $\theta_{0}>0$, there exists a constant $k\left(\theta_{0}\right)$ such that for any hyperbolic triangle with sidelengths $a, b, c$ and angle $\theta$ opposite to $c$ with $\theta \geq \theta_{0}$, we have $a+b-c<k\left(\theta_{0}\right)$. In the case where $\tilde{\eta}$ intersects $\tilde{\eta}_{Q}$, as in the figure on the left, we apply this to the triangles $\triangle o p \hat{p}$ and $\triangle o q \hat{q}$ and get

$$
l_{\sigma}(p \hat{p})+l_{\sigma}(\hat{p} \hat{q})+l_{\sigma}(q \hat{q})-l_{\sigma}(p q)<k(\pi / 2) .
$$

In the case on the right, we apply this to triangles $\triangle p \hat{q} \hat{p}$ and $\triangle p q \hat{q}$. To see that $\angle q \hat{q} p$ is bounded below by some $\theta_{0}$, observe that $\angle q \hat{q} p=$ $\pi / 2-\angle p \hat{q} \hat{p}$ and that $\sin (\angle p \hat{q} \hat{p})<1 / \cosh l_{\sigma}(\hat{p} \hat{q})$. Since $l_{\sigma}(\hat{p} \hat{q})=l_{\sigma}\left(\tilde{\eta}_{Q}\right)>$ $c(\ell)$, it follows that $\angle p \hat{q} \hat{p}$ is bounded away from $\pi / 2$ and so $\angle q \hat{q} p$ is 
bounded below by some constant $\theta_{0}=\theta_{0}(\ell)$, as desired. Thus in this case,

$$
l_{\sigma}(p \hat{p})+l_{\sigma}(\hat{p} \hat{q})+l_{\sigma}(q \hat{q})-l_{\sigma}(p q)<k(\pi / 2)+k\left(\theta_{0}(\ell)\right),
$$

completing the proof of Equation (30).

Combining Equations(29),(30),(32), we obtain

$$
\left|l_{\sigma}(\eta)-\left[l_{\sigma}\left(\eta_{Q}\right)+l_{\sigma}\left(\alpha_{q}\right) \frac{T w_{\sigma}\left(\xi, \alpha_{q}\right)}{2}+l_{\sigma}\left(\alpha_{p}\right) \frac{T w_{\sigma}\left(\xi, \alpha_{p}\right)}{2}\right]\right|<K(\ell) .
$$

Summing over all arcs $\eta$ in $\xi \cap Q$, we obtain

$$
\left|l_{\sigma}(\xi \cap Q)-\left[l_{\sigma}\left(\xi_{Q}\right)+\sum_{j} l_{\sigma}\left(\alpha_{j}\right) \frac{T w_{\sigma}\left(\xi, \alpha_{j}\right)}{2} i\left(\xi, \alpha_{j}\right)\right]\right|<K(\ell) \cdot i(\xi, \partial Q) .
$$

\section{REFERENCES}

[1] A. Beardon, Geometry of Discrete Groups, Springer Verlag (1983).

[2] L. Bers, "Uniformization, Moduli and Kleinian groups", Bull. London Maths. Soc. 4 (1972) $257-300$.

[3] L. Bers, "An inequality for Riemann surfaces", In Differential Geometry and Complex Analysis, 87 - 93, I. Chavel and H. Farkas Eds., Springer Verlag, (1985).

[4] J. Brock, R. Canary, Y. Minsky, "The classification of Kleinian surface groups II: The Ending Lamination Conjecture", arXiv:math.GT/0412006 (2004).

[5] R. Díaz and C. Series, "Limit points of lines of minima in Thurston's boundary of Teichmüller space", Alg. Geom. Top. 3 (2003) $207-234$.

[6] F. Gardiner, Teichmüller theory and quadratic differentials, John Wiley \& Sons (1987).

[7] F. Gardiner and H. Masur, "Extremal length geometry of Teichmüller space", Complex Variables 16 (1991) 209 - 237.

[8] L. Keen and C. Series, "Continuity of convex hull boundaries", Pacific J. Math. 168 (1995) $183-206$.

[9] S. Kerckhoff, "The asymptotic geometry of Teichmüller space", Topology 19 (1980) $23-41$.

[10] S. Kerckhoff, "The Nielsen Realization Problem", Ann. of Math. 117 (1983) $235-265$.

[11] S. Kerckhoff, "Lines of minima in Teichmüller space", Duke Math. J. 65 (1992) $187-213$.

[12] A. Lenzhen, "Teichmüller geodesics which do not converge", arXiv:math.GT/0511001 (2005).

[13] B. Maskit, "Comparison of extremal and hyperbolic lengths", Ann. Acad. Sci. Fenn. 10 (1985), $381-386$.

[14] H. Masur, "Two boundaries of Teichmüller space", Duke Math. J. 49 (1982), $183-190$.

[15] H. Masur and Y. Minsky, "Geometry of the complex of curves I: Hyperbolicity", Invent. Math. 138 (1999), 103-149. 
[16] H. Masur and Y. Minsky, "Geometry of the complex of curves II: Hierarchical structure", Geom. Funct. Anal. 10 (2000) 902-974.

[17] Y. Minsky, "Harmonic maps, length, and energy in Teichmüller space", $J$. Differential Geometry 35 (1992) 151-217.

[18] Y. Minsky, "Teichmüller geodesics and ends of hyperbolic 3-manifolds", Topology 32 (1993) 625-647.

[19] Y. Minsky, "Extremal length estimates and product regions in Teichmüller space", Duke Math. J. 83 (1996) 249-286.

[20] K. Rafi, "A characterization of short curves of a Teichmüller geodesic", Geometry and Topology 9 (2005) 179-202.

[21] K. Rafi, "A combinatorial model for the Teichmüller metric", arXiv:math.GT/0509584 (2005).

[22] K. Rafi, "Thick-thin decomposition of quadratic differentials", preprint (2006).

[23] C. Series, "An extension of Wolpert's derivative formula", Pacific J. of Math. 197 (2001) $223-239$.

[24] C. Series, "Limits of quasifuchsian groups with small bending", Duke Mathematical J. 128 (2005) $285-329$.

[25] K. Strebel, Quadratic Differentials, Springer Verlag (1980).

[26] S. Wolpert, "An elementary formula for the Fenchel Nielsen twist", Comment. Math. Helvetici 56 (1981) 132-135.

[27] S. Wolpert, "The length spectra as moduli for compact Riemann surfaces", Ann. of Math. 109 (1979) 323-351.

E-mail address: choiye@math.ucdavis.edu

E-mail address: rafi@math.uconn.edu

E-mail address: cms@maths.warwick.ac.uk 